\title{
Isospin Dynamics in Peripheral Heavy Ion Collisions at Fermi Energies
}

\author{
J. Rizzo ${ }^{\mathrm{a}, \mathrm{b}, \mathrm{d}}$ M. Colonna ${ }^{\mathrm{a}}$ V. Baran ${ }^{\mathrm{a}, \mathrm{b}, \mathrm{c}}$ M. Di Toro ${ }^{\mathrm{a}, \mathrm{b}}$ \\ H.H. Wolter ${ }^{\mathrm{e}, \mathrm{a}}$ M. Zielinska-Pfabe ${ }^{\mathrm{f}}$ \\ ${ }^{a}$ Laboratori Nazionali del Sud INFN, I-95123 Catania, Italy \\ ${ }^{\mathrm{b}}$ Physics and Astronomy Dept., University of Catania \\ ${ }^{\mathrm{c}}$ University of Bucharest and NIPNE-HH, Bucharest, Romania \\ ${ }^{\mathrm{d}}$ Dip. di Fisica, University of Florence, Italy \\ ${ }^{\mathrm{e}}$ Fak. f. Physik, Universität München, D-85748 Garching, Germany \\ ${ }^{\mathrm{f}}$ Smith College, Northampton, Mass. USA \\ e-mail: colonna@lns.infn.it
}

\begin{abstract}
We present a detailed study of isospin dynamics in peripheral collisions at Fermi energies. We consider symmetric and mixed collisions of ${ }^{124,112} \mathrm{Sn}$ isotopes at 35 and $50 \mathrm{AMeV}$ to study the isospin transport between the different reaction components (residues, gas and possibly intermediate mass fragments) and, in particular, the charge equilibration in the mixed system. We evaluate the effects of drift terms due to asymmetry and density gradients, which are directly related to the poorly known value and slope of the symmetry energy below saturation density. We verify the importance of an isoscalar momentum dependence of the mean field, which is found to influence the isospin transport since it changes the reaction times. We finally suggest two observables particularly sensitive to the isovector part of the nuclear equation-of-state: the correlation between isospin equilibration and kinetic energy loss for binary events, and the isospin content of the produced mid-rapidity fragments for neck fragmentation events.
\end{abstract}

Key words: charge equilibration, isospin transport coefficients, symmetry energy, kinetic energy loss, neck fragments

PACS numbers: 25.70.Lm, 25.70.Pq

Preprint submitted to Elsevier Preprint

16 November 2018 


\section{Introduction}

There has been much interest in recent years in the determination of the nuclear symmetry energy as a function of density, which is of great importance for nuclear structure as well as for astrophysical processes. While there has been much experimental and theoretical work in this direction in the last years, the symmetry energy must still be considered as uncertain. Observables, which are sensitive to the isovector density dependence of the Equation-of-State (isoEoS) and testable experimentally, are still under investigation $[1,2,3,4,5]$.

The symmetry energy $E_{\text {sym }}$ appears in the energy density

$$
\epsilon\left(\rho, \rho_{3}\right) \equiv \epsilon(\rho)+\rho E_{\text {sym }}(\rho)\left(\rho_{3} / \rho\right)^{2}+O\left(\rho_{3} / \rho\right)^{4}+\ldots,
$$

expressed in terms of total $\left(\rho=\rho_{p}+\rho_{n}\right)$ and isospin $\left(\rho_{3}=\rho_{n}-\rho_{p}\right)$ densities. The symmetry term has a kinetic contribution directly from basic Pauli correlations and a potential part from the highly controversial isospin dependence, as a function of the total nucleon density, of the mean field [4]. Both at sub-saturation and supra-saturation densities, predictions based on the existing many-body techniques diverge rather widely, see ref. [6]. However, the dominant quadratic dependence on the asymmetry parameter $\beta=\left(\rho_{3} / \rho\right)=$ $(N-Z) / A$ in Eq.(1) is well supported by the phenomenology (e.g. the mass formula) as well as by all microscopic nuclear many-body calculations [3]. We recall that the knowledge of the EoS of asymmetric matter is very important at low densities ( e.g. neutron skins, pigmy resonances, nuclear structure at the drip lines, neutron distillation in fragmentation, neutron star formation and crust) as well as at high densities ( e.g. neutron star mass-radius relation, cooling, hybrid structure, transition to a deconfined phase, formation of black holes).

It is attractive to take advantage of new opportunities in experiments (availability of very asymmetric radioactive beams, improved methods of measuring event-by-event correlations) and theory (development of reliable microscopic transport descriptions for heavy ion collision (HIC)) to obtain results which constrain the existing effective interaction models. In this paper we will discuss dissipative collisions in the range of Fermi energies, which will yield information on the symmetry term around and below normal density. Here we focus our attention on the charge equilibration dynamics in peripheral collisions where we expect to see symmetry energy effects on the isospin transport. The interesting feature at Fermi energies is the onset of collective flows due to compression and expansion of the interacting nuclear matter. The isospin transport takes place in regions with density and asymmetry variations and thus we expect to have contributions to the isospin current from charge and mass drift mechanisms. It was shown [7], that these are determined by the 
value and the density-gradient of the symmetry energy, respectively [7], which are then studied here at subsaturation densities. Fast (pre-equilibrium) nucleon emission, related to the symmetry energy via the different symmetry potentials seen by neutrons and protons, also influences the isospin content of the Projectile-like and Target-like residues. Finally in the Fermi energy range we have a substantial Intermediate Mass Fragment (IMF) production even in non-central collisions and this will also affect the isospin dynamics. We will carefully look at all these dynamical effects, trying to select observables which are sensitive to the density dependence of the symmetry term.

Isospin equilibration at intermediate energies and its relation to the symmetry energy has, in fact, attracted much attention in recent years in experiment as well as in theory $[7,8,9,10,11]$. We present here a detailed analysis of the various iso-transport mechanisms in connection to properties of the effective in-medium interactions. We try to disentangle effects due to the general reaction dynamics, ruled by isoscalar terms of the interaction, from genuine isovector contributions. This will allow us to suggest new observables particularly sensitive to the symmetry term of the EoS:

(i) Correlation between the isospin equilibration of the reaction partners and the total kinetic energy loss in binary events;

(ii) The isospin content of IMF in break-up (ternary) events.

In Sect.2 we introduce our transport-theoretical approach to the reaction dynamics and the choice of the effective interactions investigated. In Sect.3 we show results for binary events in $S n+S n$ peripheral collisions at $50 \mathrm{AMeV}$, in Sect. 4 we discuss neck fragmentation events, and finally in Sect.5 we present a summary and some perspectives.

\section{Theoretical Description of the Collision Dynamics}

\subsection{The Transport Model}

We perform ab initio collision simulations using the microscopic Stochastic Mean Field (SMF) model. It is based on mean field transport theory with correlations included via hard nucleon-nucleon (NN) collisions and with inclusion of stochastic forces acting on the mean phase-space trajectory $[4,12,13,14,15]$. Stochasticity is essential in order to allow the growth of dynamical instabilities with fragment production, as well as to obtain physical widths of distributions of observables. Moreover it will allow to perform event-by-event correlation studies of great importance for the very complex reaction dynamics in this energy regime. 
The transport equation for the phase space distribution function, with the Pauli blocking consistently evaluated, is integrated following a representation in terms of test particles of finite widths [12,13]. A detailed description of the procedure is given in ref. [4]. Our code [16] has been extended by the introduction of momentum dependent mean fields (see next subsection), which are rather important in this energy range. It has also been possible to improve the numerical accuracy while even reducing the computing times [17].

A parametrization of free nucleon-nucleon cross sections is used, with isospin, energy and angular dependence [18]. Low energy NN collisions, mostly forbidden because of the Pauli blocking, have large cross sections and could induce spurious effects in the presence of some numerical inefficiency in the blocking procedure, due to the discretization of the phase space. In order to avoid such problems a cutoff value $\sigma_{c u t}=50 \mathrm{mb}$ is used in our calculations. A parallel ensemble method is employed in the implementation of the collision term.

For discussions of isospin dynamics in this energy regime it is essential to have a reliable procedure for fragment recognition, i.e. to identify the "gas" (emitted nucleons and light clusters) and the "liquid" (fragments, residues) phases. Fragments are produced via instabilities in the expanding dilute matter and as residues of the initial colliding ions (in the case of non-central collisions). In our transport simulations the fragment definition is performed by means of density cuts ; more precisely, a criterion of $\rho>\rho_{\text {cut }}$ (with $\rho_{\text {cut }}$ between $\rho / 6$ and $\rho / 10)$ for the liquid, and correspondingly for the gas, is used. This procedure is applied in an event by event analysis at the "freeze-out", i.e. when the resulting fragments are well separated in space and interacting only via Coulomb forces. We have checked that such "final" gas/liquid isospin properties are not depending on the choice of density cuts and freeze-out times, see Sect.3.2. Nucleons can be emitted during the early stages of the reaction due to hard NN collisions as well as to repulsive potentials, during the fragment formation due to the isospin distillation effect, and finally from sequential decay of excited primary products (fragments/residues). The isospin is used in all these dynamical paths both as a tracer of the reaction mechanism, as well as an observable of interest with respect to the iso-EoS.

We follow the reaction dynamics only up to a "freeze-out" time. Fragments and residues will still be highly excited and will undergo sequential decays that can modify the original isospin information related to the nuclear dynamics. However, we will analyze mainly quantities which are expected to change little due to the late evaporative emission. "Imbalance" and "Double" Ratios have been suggested to that purpose $[8,19,20]$, and will be discussed below. 


\subsection{Specification of Interactions}

We adopt a generalized form of effective interaction, which can be easily reduced to Skyrme-like forces, with momentum dependent terms also in the isovector channel $[17,21,22]$. The general structure of this isoscalar and isovector Momentum Dependent (MD) effective interaction is derived via an asymmetric extension of the Gale-Bertsch-DasGupta (GBD) force [23,24,25,26], which corresponds to a Yukawian non-locality. The energy density is parametrized as follows:

$$
\varepsilon=\varepsilon_{k i n}+\varepsilon_{A}\left(A^{\prime}, A^{\prime \prime}\right)+\varepsilon_{B}\left(B^{\prime}, B^{\prime \prime}\right)+\varepsilon_{C}\left(C^{\prime}, C^{\prime \prime}\right),
$$

where $\varepsilon_{k i n}$ is the usual kinetic energy density and the potential terms are

$$
\begin{aligned}
& \varepsilon_{A}\left(A^{\prime}, A^{\prime \prime}\right)=\left(A^{\prime}+A^{\prime \prime} \beta^{2}\right) \frac{\varrho^{2}}{\varrho_{0}} \\
& \varepsilon_{B}\left(B^{\prime}, B^{\prime \prime}\right)=\left(B^{\prime}+B^{\prime \prime} \beta^{2}\right)\left(\frac{\varrho}{\varrho_{0}}\right)^{\sigma} \varrho \\
& \varepsilon_{C}\left(C^{\prime}, C^{\prime \prime}\right)=C^{\prime}\left(\mathcal{I}_{N N}+\mathcal{I}_{P P}\right)+C^{\prime \prime} \mathcal{I}_{N P}
\end{aligned}
$$

The variable $\beta=(N-Z) /(A)$ defines the isospin content or asymmetry of the system, given the number of neutrons $(N)$, protons $(Z)$, and the total mass $A=N+Z$; $\varrho$ is the nuclear matter density ( $\varrho_{0}$ is the saturation value). The momentum dependence is contained in the $\mathcal{I}_{\tau \tau^{\prime}}$ terms, which are integrals of the form

$$
\mathcal{I}_{\tau \tau^{\prime}}=\int d \vec{p} d \vec{p}^{\prime} f_{\tau}(\vec{r}, \vec{p}) f_{\tau^{\prime}}\left(\vec{r}, \vec{p}^{\prime}\right) g\left(\vec{p}, \vec{p}^{\prime}\right),
$$

with $\tau=P, N$, for protons and neutrons. Here $f_{\tau}(\vec{r}, \vec{p})$ are the nucleon phase space distributions for protons and neutrons and the function $g\left(\vec{p}, \vec{p}^{\prime}\right) \equiv$ $g\left[\left(\vec{p}-\vec{p}^{\prime}\right)^{2}\right]$ determines the type of momentum dependence. A Skyrme-like momentum dependence is obtained when we use the simple quadratic form $g\left(\vec{p}, \vec{p}^{\prime}\right)=\left(\vec{p}-\vec{p}^{\prime}\right)^{2}$. A more general momentum dependence, in better agreement with phenomenological optical potentials, can be introduced by the function $[23,24,25,26]$

$$
g\left(\vec{p}, \vec{p}^{\prime}\right)=\left[1+\left(\frac{\vec{p}-\vec{p}^{\prime}}{\Lambda}\right)^{2}\right]^{-1}
$$

We remark that this form is particularly suitable for $S M F$ simulations. 
From the energy density one derives the mean field potentials as $U_{\tau}(\vec{r}, \vec{p})=$ $\delta \varepsilon / \delta f_{\tau}$. Thus the above energy density implies a momentum dependent mean field interaction. The momentum dependence is isoscalar if the coefficients $C^{\prime}$ and $C^{\prime \prime}$ are identical and also it can have an isovector part, if they are different. The isovector momentum dependence implies different effective masses for protons and neutrons given as $\frac{m_{\tau}^{*}}{m}=\left(1+\frac{m}{\hbar^{2} p} \frac{\partial U_{\tau}}{\partial p}\right)^{-1}$.

We note that the used form of the Momentum Dependent effective interaction, Eqs.(2 - 5), is equivalent to the $M D I$ force introduced in ref.[26]. The choice of the parameters is different: having fixed the isoscalar properties of the EoS (soft symmetric matter $K_{N M}\left(\rho_{0}\right)=215 \mathrm{MeV}$, with nucleon effective mass $\left.m^{*} / m=0.67\right)$, we are exploring the dynamical effects of a different density dependence of the symmetry term, isovector part of the EoS, see Eq.(1). In Table I we report the used parametrization.

Tab. I: Parameters of the Momentum Dependent (MD) Interaction.

\begin{tabular}{c|c|c|c|c} 
Isoscalar & & Isovector & Asy-stiff & Asy-soft \\
\hline$A^{\prime}$ & $-55.626 \mathrm{MeV}$ & $A^{\prime \prime}$ & $-41.312 \mathrm{MeV}$ & $59.742 \mathrm{MeV}$ \\
\hline$B^{\prime}$ & $63.013 \mathrm{MeV}$ & $B^{\prime \prime}$ & $29.826 \mathrm{MeV}$ & $-71.247 \mathrm{MeV}$ \\
\hline$C^{\prime}$ & $-129.41 \mathrm{MeV}$ & $C^{\prime \prime}$ & $-1354.78 \mathrm{MeV}$ & $-1354.78 \mathrm{MeV}$ \\
\hline$\sigma$ & 1.242 & & & \\
\hline$\Lambda$ & $2.106 \mathrm{fm}^{-1}$ & & & \\
\hline
\end{tabular}

Here we want to test the sensitivity of isospin transport observables to two essentially different behaviors of the symmetry energy around saturation: asysoft with a smoothly decreasing behavior below saturation, and asy-stiff with instead a rapid decrease to lower densities [1,4]. In Fig.1 we show the density dependence for these two typical choices. We remark that this is the total symmetry energy, with both potential and kinetic contributions.

At Fermi energies we have substantial density variations during the collision. In particular during the expansion phase the system probes more dilute regions. Then we expect important effects on the isospin transport due to different subsaturation values (larger for asy-soft) and to different slopes (larger for asy-stiff) of the symmetry energy. We will see that the transport coefficients due to density and isospin gradients (usually called "drift", resp. "diffusion" coefficients) will be affected in a different way and the final result will be sensitive to the choice of the two $E_{\text {sym }}$ shown in Fig.1.

In the framework of our parametrization of the effective interaction we can also easily adjust the isospin momentum dependence to have the same den- 


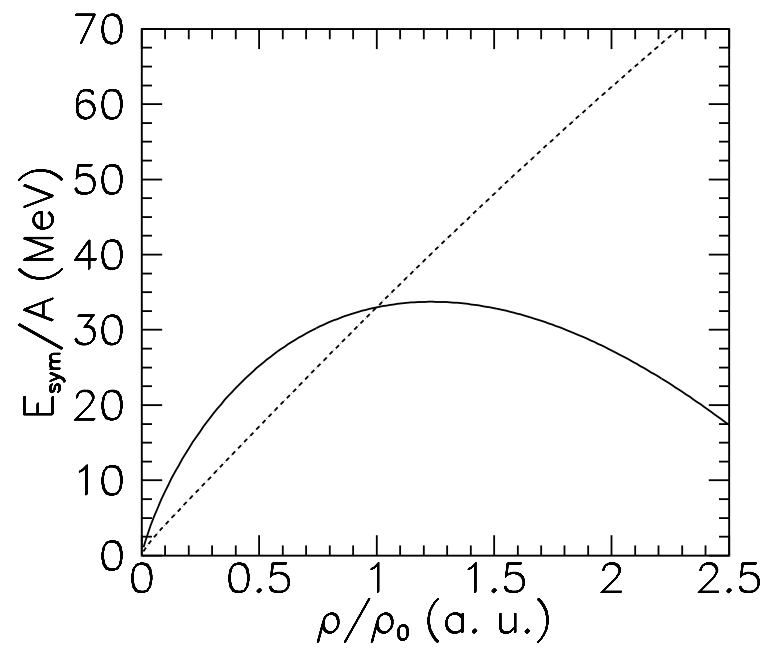

Fig. 1. Density dependence of the symmetry energies used in the simulations presented here: asy-soft (solid) and asy-stiff (dashed).

sity dependence of the symmetry energy but opposite neutron/proton effective mass splittings. The ordering of the neutron/proton effective masses, which is also related to the slope of the Lane potential with energy, is much debated in the literature $[4,6]$. E.g. one could have $m_{n}^{*}>m_{p}^{*}$ as predicted by the early Skyrme forces [27] or the opposite as for the later Skyrme-Lyon parametrizations [28]. Thus one can, separately from the density dependence, study the corresponding dynamical effects on nucleon emissions [17,21,22].

The effective mass splitting is increasing with density and it is relevant for the dynamics mainly at high momenta. Indeed, it has been shown that the effect on nucleon emission and flows can be observed only at high transverse momenta $[17,21,22]$. At the energies investigated here it is, however, a secondary effect, as the transport phenomena are mainly driven by the value and the slope of the symmetry energy below saturation density. Thus, in order to simplify the presentation, we have fixed the isovector momentum dependence. The results presented here are obtained with the choice $m_{n}^{*}>m_{p}^{*}$. We have actually verified that the influence of the different mass splittings on the present results on isospin transport is very small.

\subsection{Symmetry Potentials}

Many investigations have given definite evidence that the effective mean fields are momentum dependent, i.e. have an important isoscalar momentum dependence (see e.g. ref. [29]). Only in this way it was possible to explain the dependence with energy of the nucleon flow in heavy collisions. We have then 


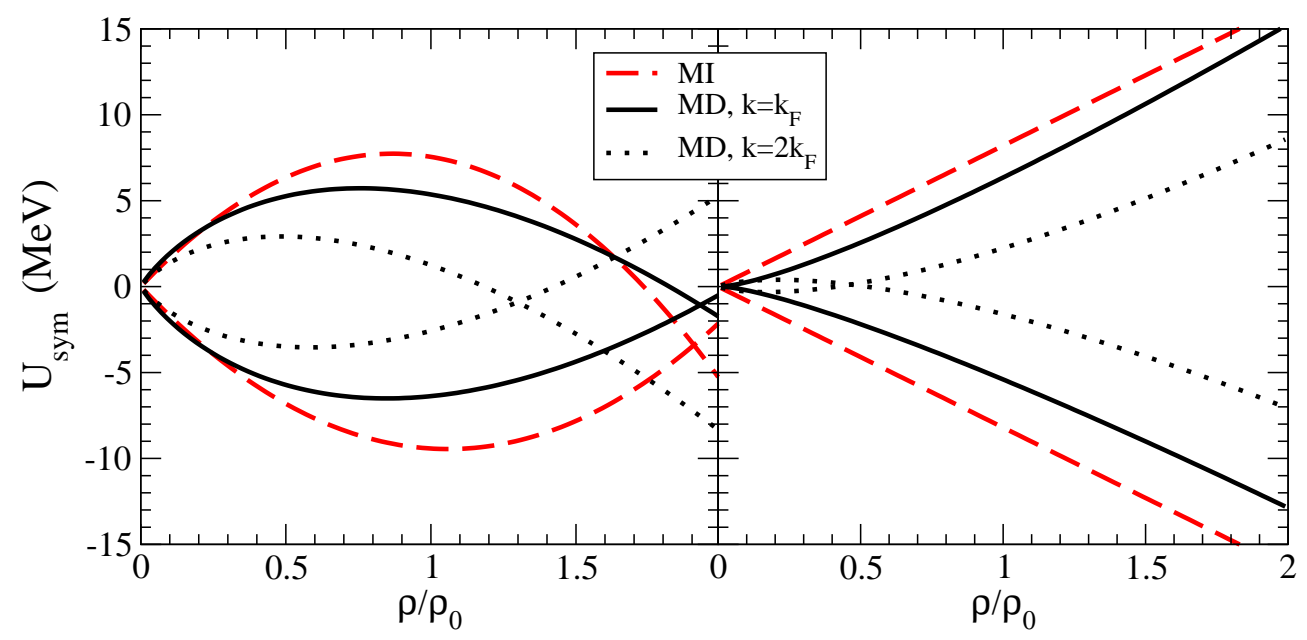

Fig. 2. Symmetry potentials for neutrons (upper curves) and protons (lower curves) in a matter with asymmetry $\beta=0.2\left({ }^{124} \mathrm{Sn}\right.$-like). Left panel: asy-soft choice; right panel: asy-stiff choice; MI interaction: dashed lines, MD interaction: solid $\left(k=k_{F}\right)$ and dotted $\left(k=2 k_{F}\right)$ lines

naturally included a momentum dependence of the isoscalar interaction adjusted to these data, as discussed before. However, it is still of interest to see the effects of such isoscalar momentum dependence on isospin transport phenomena, since this has also been an issue in earlier calculations [11], and has not been looked at systematically so far. Thus in the following we will also show results with Momentum Independent (MI) interactions along with the Momentum Dependent (MD) ones, of course with parameters adjusted to the same saturation properties and symmetry terms. Details about the MI interaction can be found in ref. [4].

We remark that the corresponding symmetry potentials, i.e. the effective field variations seen by neutrons and protons in asymmetric matter, can be rather different in the two frames, MI vs MD, and this could affect the isospin transport properties. So this point deserves some more attention.

Just to have an idea of the expected transport effects we plot in Fig.2 the symmetry potentials for asymmetry matter with $\beta=0.2$, the mean asymmetry of a ${ }^{124} \mathrm{Sn}$ isotope. We note that the momentum dependence, in fact, modifies the effect of the stiffness of the symmetry term on the nucleon potentials. E.g. comparing the asy-soft vs. asy-stiff choices for neutrons and for MI interactions, we see the general trend of a larger repulsion below saturation and a larger attraction above $\rho_{0}$ (and opposite for protons). It is also seen, that the effect can be different in the MD case, though depending strongly on the nucleon momenta. In this respect we remark that, in particular below the saturation density, the differences become appreciable for nucleon momenta around $2 k_{F}$, not much present in the Fermi energy range of interest here. 

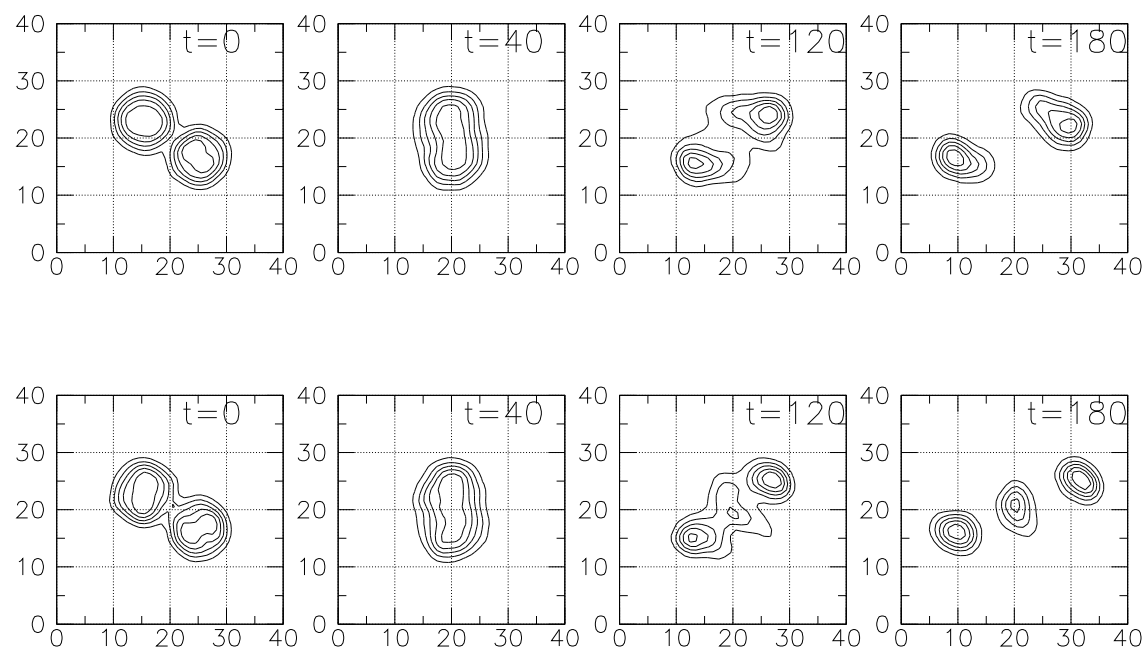

Fig. 3. Density contours of a binary (upper panel) and a ternary (lower panel) event in the simulation of ${ }^{124} \mathrm{Sn}+{ }^{124} \mathrm{Sn}$ semiperipheral $(b=6 \mathrm{fm})$ collisions at $50 \mathrm{AMeV}$. A momentum dependent interaction $(M D)$ has been used.

At variance a relevant overall effect of the Momentum Dependence is the reduction of the interaction times during the collision with an expected clear influence on the isospin diffusion. An important consequence is that in order to extract information on the density dependence of the symmetry term we are forced to select new observables related to the isospin equilibration that should be not very sensitive to isoscalar properties of the effective interactions, like the main momentum dependence. We will discuss in detail this point in the next sections, suggesting very promising possibilities.

\section{General Features of the Isospin Transport in Peripheral Colli- sions}

In this work we investigate peripheral collisions between similar systems with different isospin, specifically collisions of different combinations of $S n$ isotopes. In Fig.3 density contours of two typical examples of such events are shown, one in the upper panel resulting in two heavy fragments and nucleons or light clusters (binary event), and one in the bottom panel producing a third fragment of intermediate mass from the neck region between the two heavy residues (ternary event, neck fragmentation ). We will discuss binary events in Sect. 4, especially with respect to isospin equilibration between the residues, and ternary events in Sect. 5 with respect to the isospin content of the neck fragments. 


\subsection{Phases of the Reaction Mechanism}

The reaction mechanism can be divided into different phases, which will be important for the following discussion:

(i) Pre-equilibrium phase: the approach and early interaction phase is characterized by an emission of particles and light clusters (pre-equilibrium emission), which contributes to the "gas" phase. Of interest is the asymmetry of the gas, which has recently been discussed in detail in central collisions in similar systems, in particular, with respect to ratios of produced particles and fragments $[19,20]$.

(ii) Transport phase: a neck of density below normal values develops between the two heavy residues, the evolution of which is driven by the motion of the spectators. During this phase isospin is transferred to the neck due to the density difference between the neck and the residues; this effect has been called isospin migration (isospin drift), which leads to a more neutron-rich neck [30,31]. Moreover, in collision systems with different asymmetry, isospin is also transported through neck due to the $N / Z$ "concentration" difference, leading to an equilibration of the isospin of the residues (projectile- and target-like fragments; PLF/TLF). This has been called isospin diffusion. In asymmetric systems there is a competition of isospin migration and diffusion, which contains information on the characteristic time scales of the processes.

(iii) Fragmentation into identified fragments (primary fragments): as in Fig.3 we distinguish two types of events, binary events with two heavy residues and the gas, and "ternary" events with an additional fragment of charge $Z \geq 3$ and with mass between the masses of the residues and the gas, i.e. an intermediate mass fragment (IMF). Events with several IMF's are very rare at these energies and impact parameters and will not be considered.

(iv) Evaporation stage: the primary fragments are considerably excited and deexcite on the way to the detector, also changing their asymmetry. This part is not included in our dynamical model, but could rather be accessed with statistical evaporation codes. This is not done here, because we try to use observables, which are expected not to change substantially in the secondary evaporation. It should be kept in mind, however, that the experimental "gas" also contains contributions from the evaporation stage.

\subsection{Freeze-out Times and Density Cuts}

In the next sections we will present a detailed study of the isospin structure of the reaction products analyzed at the freeze-out times, i.e. of the "primary" 

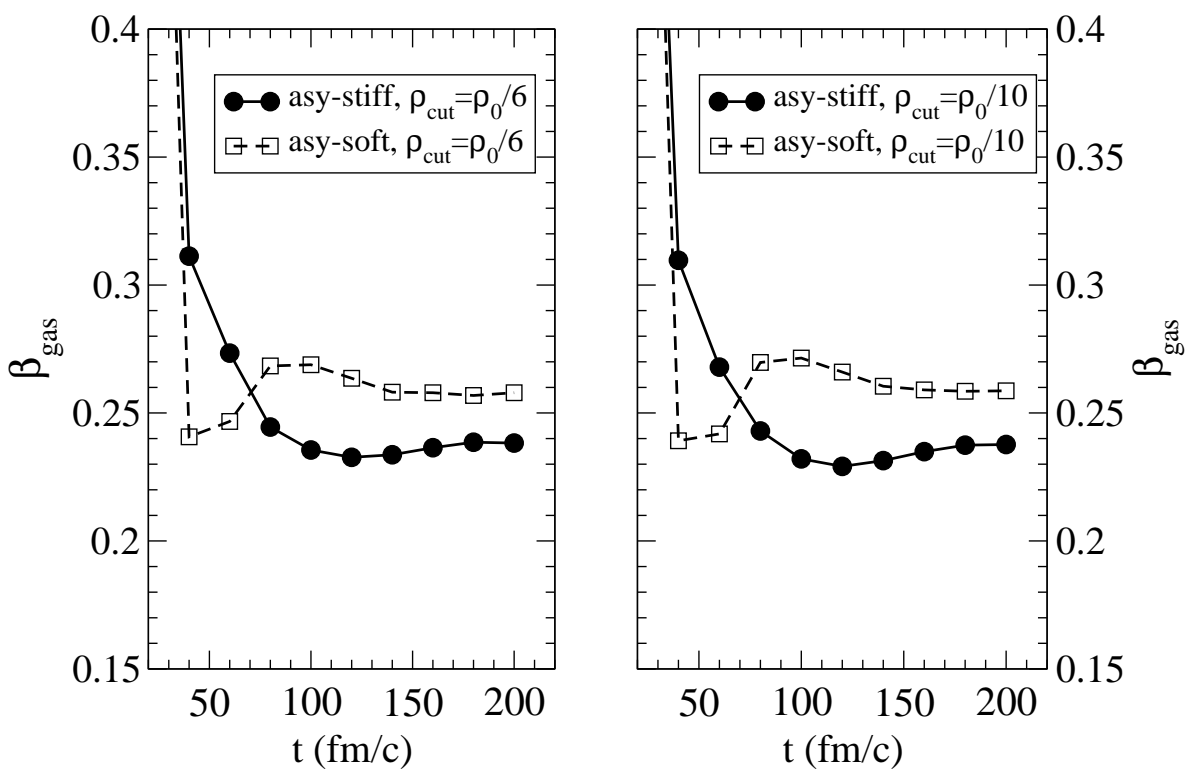

Fig. 4. Time evolution of the "gas" asymmetry in the simulation of ${ }^{124} S n+{ }^{124} S n$ semiperipheral $(b=6 \mathrm{fm})$ collisions at $50 \mathrm{AMeV}$. Asy-soft: dashed lines. Asy-stiff: solid lines. The panels correspond to different density cuts for the "gas" definition, see text. A momentum dependent interaction $(M D)$ has been used.

nuclear systems (nucleons, clusters and fragments) emerging from the reaction region. Here we would like to show that the extracted isospin information is reliable, i.e. not much dependent on not well defined parameters, like the choice of the freeze-out times and of the density cuts needed to separate the liquid and gas phases.

In Fig. 4 we show the time evolution of the "gas" asymmetry averaged over 200 event simulations of the ${ }^{124} \mathrm{Sn}+{ }^{124} \mathrm{Sn}$ semiperipheral $(b=6 \mathrm{fm})$ collisions at $50 \mathrm{AMeV}$, with the two choices of the symmetry stiffness (MD interactions). From the density contour plots of Fig.3 we see that the freeze-out time should be between 150 and $200 \mathrm{fm} / \mathrm{c}$. The flat behaviour of the gas asymmetry after $150 \mathrm{fm} / \mathrm{c}$ clearly indicates that this quantity will not be much sensitive to a freeze-out choice in that interval. Of course the same will happen to the liquid phase. The calculation has been performed using a $\rho_{0} / 6$ (left panel) and a $\rho_{0} / 10$ (right panel) density cut for the gas phase. We can see that almost identical results can be obtained. An interesting point to note is the larger final gas asymmetry with the asy-soft choice. This is a combined effect of the neutron distillation during the fragment formation (which gives the crossing at around $70 \mathrm{fm} / \mathrm{c}$ ) and of the larger repulsive potential below saturation, see Fig.2.

Tab. II: ${ }^{124} S n+{ }^{124} S n$ collisions: Freeze-out times (fm/c) vs. beam energy, centrality and momentum dependence. 


\begin{tabular}{l|c|c|c|c|c|c|c}
$35 \mathrm{AMeV}$ & $\mathrm{b}(\mathrm{fm})$ & $\mathrm{MD}$ & $\mathrm{MI}$ & $50 \mathrm{AMeV}$ & $\mathrm{b}(\mathrm{fm})$ & $\mathrm{MD}$ & $\mathrm{MI}$ \\
\hline & 6 & 180 & 200 & & 6 & 140 & 150 \\
\hline & 8 & 160 & 175 & & 8 & 120 & 125 \\
\hline & 10 & 120 & 125 & & 10 & 100 & 100 \\
\hline
\end{tabular}

Finally in Table II we give our quantitative estimates of the variation of the freeze-out times with beam energy, centrality and momentum dependence of the used effective interactions. The nuclear interaction times are different for MD and MI interactions (shorter for MD) and this property of the isoscalar dynamics will certainly affect the isospin diffusion. In order to extract the role of the isovector part of the interaction we have to single out observables directly related to the interaction times.

\subsection{Isospin Transport Coefficients}

As already pointed out, in heavy ion collisions at Fermi energies isospin transport is due to the combined effect of asymmetry and density gradients. It can be discussed in a compact way by means of the chemical potentials for protons and neutrons as a function of density $\rho$ and isospin asymmetry $\beta$ [7]. In fact, the $p / n$ currents can be expressed as

$$
\mathbf{j}_{p / n}=D_{p / n}^{\rho} \nabla \rho-D_{p / n}^{\beta} \nabla \beta
$$

with $D_{p / n}^{\rho}$ the mass, and $D_{p / n}^{\beta}$ the isospin transport coefficients, which are directly given by the variation of $n, p$ chemical potentials with respect to density and asymmetry, see ref. [7]. In the literature these are often referred to as the drift, resp. diffusion coefficients, and we will follow this convention here. Of interest here is the differential current of neutrons and protons (iso-vector current) which has a simple relation to the density dependence of the symmetry energy. In fact, from the rather general parabolic form of the symmetry term in the energy density, we obtain the important relation $\mu_{n}-\mu_{p}=4 \beta E_{\text {sym }}$ and derive the drift and diffusion contributions to the isovector current

$$
\begin{aligned}
& D_{n}^{\rho}-D_{p}^{\rho} \propto 4 \beta \frac{\partial E_{\text {sym }}}{\partial \rho}, \\
& D_{n}^{\beta}-D_{p}^{\beta} \propto 4 \rho E_{\text {sym }} .
\end{aligned}
$$

Thus the isospin transport due to density gradients, i.e. isospin migration, depends on the slope of the symmetry energy, or the symmetry pressure, while the transport due to isospin gradients, i.e. isospin diffusion, depends on 
the absolute value of the symmetry energy. In peripheral collisions discussed here, residues of about normal density are in contact with the neck region of density below saturation density. In this region of density a stiff iso-EoS has a smaller value but a larger slope compared to a soft iso-EoS. Correspondingly we expect opposite effects of these models on the migration and diffusion of isospin.

In our investigation we will see that the asy-soft choice appears to be more effective for isospin equilibration. This is the result of a dominant diffusion mechanism for peripheral collisions, together with a stronger fast neutron emission, which also contributes to the $N / Z$ equilibration. With respect to the pre-equilibrium emission we recall that in the asy-soft case neutrons see a more repulsive symmetry potential around saturation, see Section 2.3. On the other hand, the asy-stiff choice is more effective for isospin migration, which will be seen to be important for the isospin content of the neck fragments.

\subsection{The Imbalance Ratio}

We will discuss the asymmetries of the various parts of the reaction system (gas, PLF/TLF's, and in the case of ternary events, IMF's ). In particular, we study the so-called imbalance ratio (also called Rami or transport ratio [32]), which is defined as

$$
R_{P, T}^{x}=\frac{2\left(x^{M}-x^{e q}\right)}{\left(x^{H}-x^{L}\right)}
$$

with $x^{e q}=\frac{1}{2}\left(x^{H}+x^{L}\right)$. Here, $x$ is an isospin sensitive quantity that is to be investigated with respect to equilibration. In this work we consider primarily the asymmetry $\beta=(N-Z) /(N+Z)$, but also other quantities, such as isoscaling coefficients, ratios of production of light fragments, etc, can be of interest [5]. The indices $H$ and $L$ refer to the symmetric reaction between the heavy ( $n$-rich) and the light ( $n$-poor) systems, while $M$ refers to the mixed reaction. $P, T$ denote the rapidity region, in which this quantity is measured, in particular the PLF and TLF rapidity regions. Clearly, this ratio is \pm 1 in the projectile and target regions, respectively, for complete transparency, and oppositely for complete rebound, while it is zero for complete equilibration.

In a simple model we can show that the imbalance ratio mainly depends on two quantities: the strength of the symmetry energy and the interaction time between the two reaction partners. Let us take, for instance, the asymmetry $\beta$ of the PLF (or TLF) as the quantity $x$. In the Fermi energy domain we can describe the charge equilibration dynamics as an overdamped dipole oscillation mode, see [33,34]. Therefore, as a first order approximation, in the mixed 
reaction the charge asymmetry parameter will show an exponential relaxation towards its complete equilibration value, $\beta_{e q}=\left(\beta_{H}+\beta_{L}\right) / 2$ as

$$
\beta_{P, T}^{M}=\beta^{e q}+\left(\beta^{H, L}-\beta^{e q}\right) e^{-t / \tau},
$$

where $t$ is the time elapsed while the reaction partners are interacting (interaction time) and the damping $\tau$ is mainly connected to the strength of the symmetry energy. In fact, as seen in Eq.(7), isospin diffusivity is directly proportional to the symmetry energy $[7,10]$. Inserting this expression into Eq.(8), one obtains $R_{P, T}^{\beta}= \pm e^{-t / \tau}$ for the PLF and TLF regions, respectively.

From this simple result one sees that the imbalance ratio does not depend on the difference of asymmetries $\left(\beta_{H}-\beta_{L}\right)$ of the systems considered, at least at a first order level, which is due essentially to the normalization to the difference $\left(\beta_{H}-\beta_{L}\right)$ in the definition of $R$ in Eq.(8). Hence the imbalance ratio can be considered as a good observable to trace back the strength of the symmetry energy from the reaction dynamics. We will show that the effect of different iso-EoS choices on the final imbalance ratios are measurable, in particular, when correlated to the total energy loss of the dissipative collision, which sets the time-scale of the process.

\section{Binary Reactions}

In this section we discuss binary reaction events, and investigate the asymmetries of the various reaction components, i.e. of the gas, the residues, and the exchanged particles, as well as the imbalance parameter derived from these via Eq.(8). We will consider these quantities for the different isovectorEoS's, and for momentum-dependent (MD) and momentum-independent (MI) parametrizations of the isoscalar part, see Sect. 2.2. The aim of the analysis is also to show that effects due to the Momentum-Dependent term of the nuclear interaction are relevant for the reaction mechanism at Fermi energies, and consequently for the isospin equilibration. Here we discuss these quantities as a function of the incident energy and impact parameter, in the Sect.4.3 we will introduce the more significant correlation to the kinetic energy loss.

\subsection{Asymmetries of Reaction Components}

We first investigate the behaviour of the asymmetries $\beta$ of the reaction components for the different $S n+S n$ reactions for the impact parameters $b=6,8$, and $10 \mathrm{fm}$ and for two incident energies. We present our results for an incident energy of $E_{l a b}=50 \mathrm{AMeV}$ for the gas phase in Fig.5, for the residues in Fig.6, 


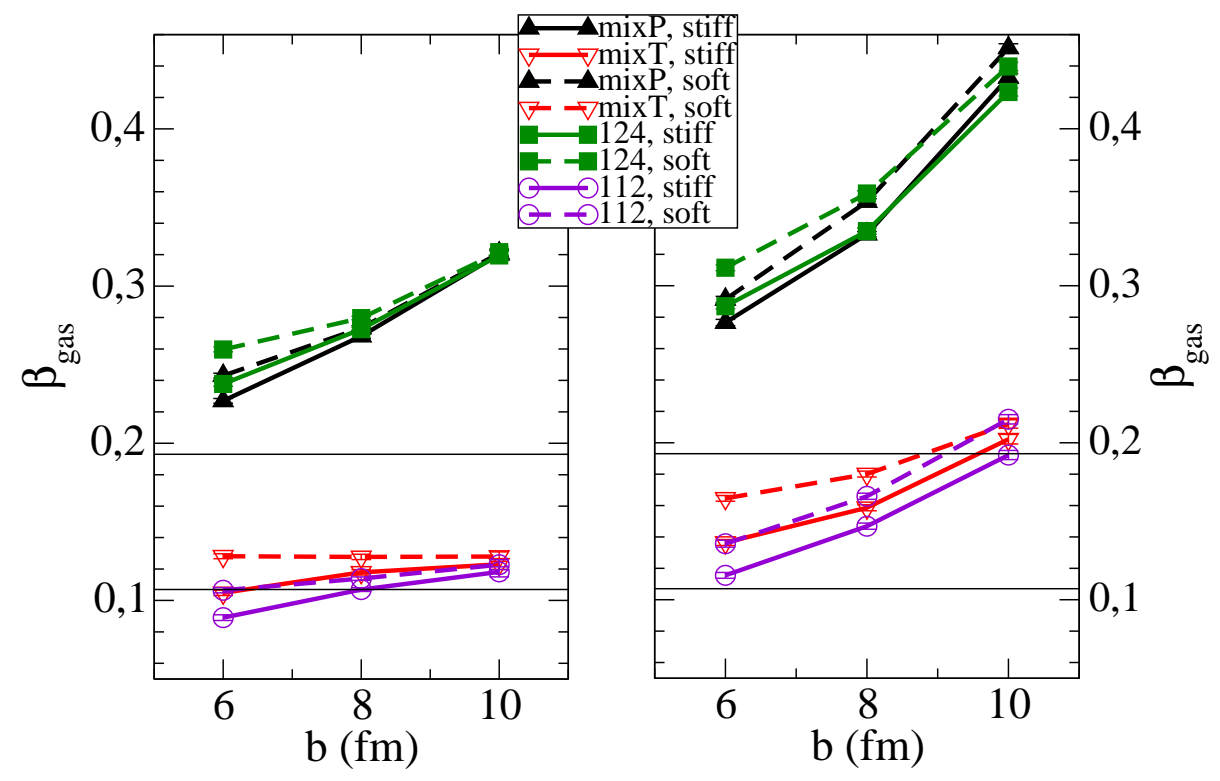

Fig. 5. Asymmetries of the gas in $S n+S n$ collisions at incident energy of $E=50 \mathrm{AMeV}$ for MD (left) and MI (right) interactions for a stiff (solid lines) and soft (dashed lines) iso-EoS. The symbols differentiate the different reaction systems: mixed PLF (full triangle up), mixed TLF (empty triangle down), symmetric heavy (full squares), symmetric light (empty, circles ). Thin horizontal lines denote the initial asymmetries of the heavy and light system, respectively.

and for the exchanged particles in Fig.7. These figures are arranged in an analogous way: the results with the momentum dependent interaction (MD) are in the left panel, those with the momentum-independent one (MI) on the right. Results for the stiff and soft iso-EoS are given by solid and dashed lines, respectively. The symbols distinguish the different systems: mixed $H L$ reaction PLF (triangle up, full), TLF (triangle down, empty), symmetric heavy $H H$ (squares, full) and light $L L$ ( circles, empty) systems. Thus, also for the gas we have separated the emitted particles according to their origin from the projectile $(\mathrm{P})$ or target $(\mathrm{T})$. The asymmetries of the exchanged particles are only given for the mixed system. The initial asymmetries of ${ }^{124} \mathrm{Sn}$ and ${ }^{112} \mathrm{Sn}$ are indicated as horizontal lines.

We first note the following general properties of these asymmetries, which will be valid for all cases discussed in the following. Of course, all these asymmetries are connected by charge conservation. In absolute numbers the mass of the exchanged particles changes from about 20 to $5 \mathrm{amu}$, and for the gas from about 40 to $20 \mathrm{amu}$, for $b=6$ to $10 \mathrm{fm}$, respectively.

- The gas (Fig. 5) is generally more $n$-rich (i.e. asymmetric) than the initial 


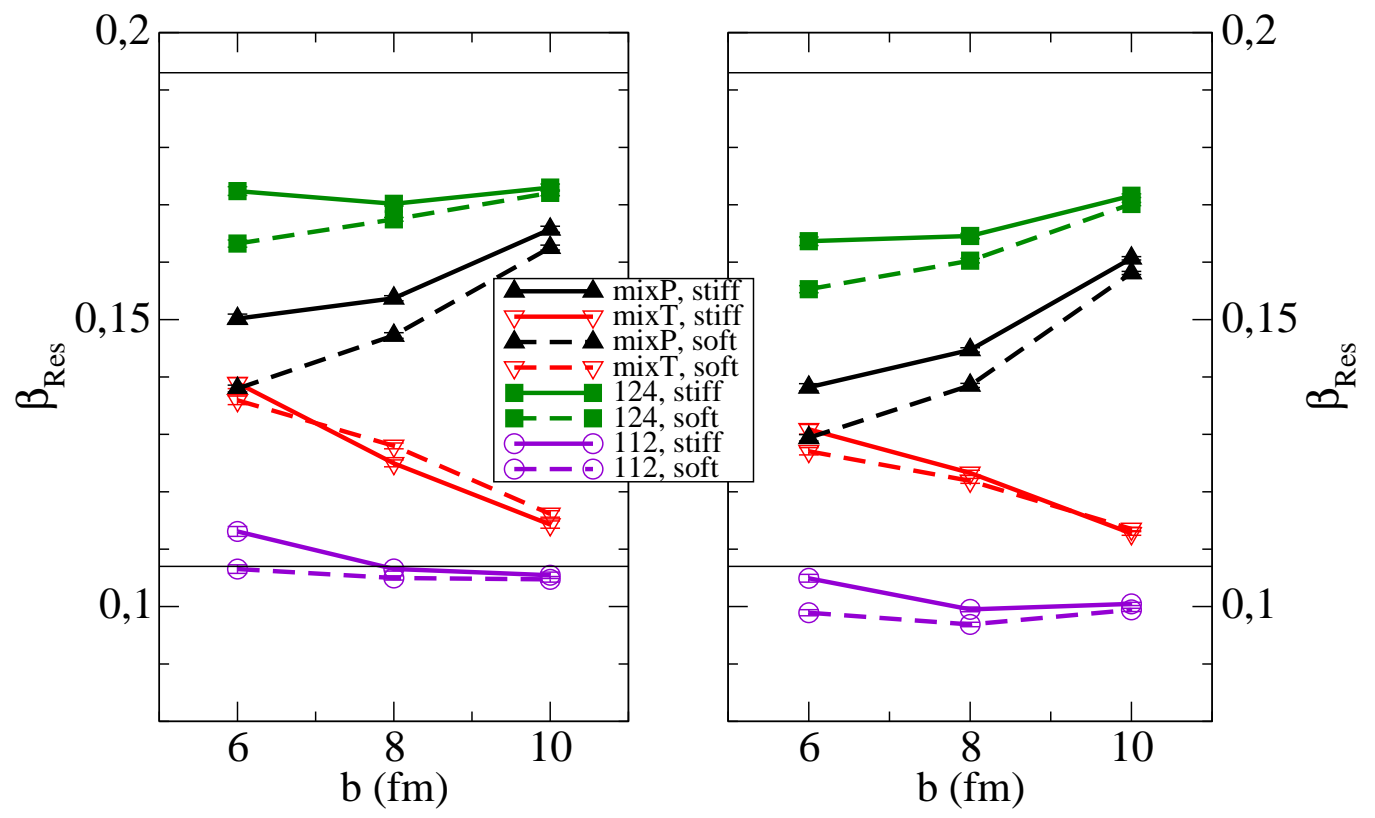

Fig. 6. Asymmetries of the residues in $S n+S n$ collisions at incident energy of $E=50 \mathrm{AMeV}$ for MD (left) and MI (right) interactions. The arrangement of the figure and the meaning of the lines and symbols is as in Fig.5.

asymmetry of the corresponding nucleus; always for the $n$-rich nucleus (P) but mostly also for the $n$-poor (less $n$-rich) system (T). In a nuclear medium with neutron excess the interaction is more repulsive for neutrons, such that these are emitted preferentially.

- The asymmetry of the residues (Fig.6) for the mixed $H L$ system decreases for the $n$-rich (PLF) and increases for the $n$-poor (TLF) partner with respect to the initial asymmetries, as expected for isospin equilibration. It is interesting to look closer at the residue asymmetry $\beta_{\text {Res }}$ in the case of the $H H$ and $L L$ symmetric collisions. Here we cannot have isospin transport and the only variations come from nucleon emissions. The asymmetry decreases for the symmetric systems from the initial asymmetry, less evident in the $n$-poor system, in correspondence with the asymmetry of the gas. The change in the asymmetries of the residues of the mixed $H L$ systems is much bigger than in the symmetric systems, indicating the importance of the transport contributions to the isospin current. This is also supported by the dependence on the iso-EoS discussed below.

- In the mixed $H L$ system neutrons are predominantly exchanged from the $n$-rich to the $n$-poor system, i.e. the asymmetry of the exchanged particles in this direction is much larger than the initial asymmetry of the $n$-rich system. In the other direction the asymmetry is close to the initial asymmetry. 

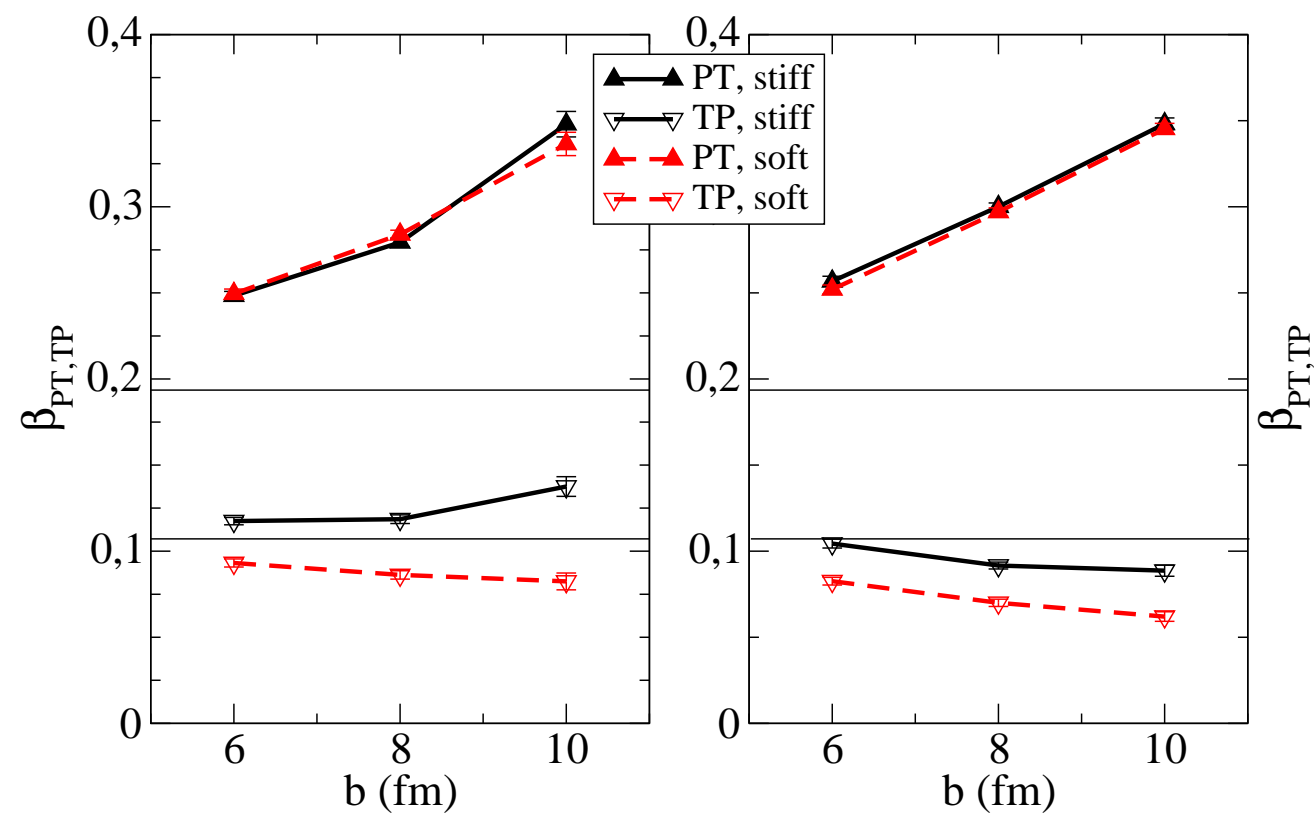

Fig. 7. Asymmetries of the exchanged particles in the collision ${ }^{124} S n+{ }^{112} \mathrm{Sn}$ at incident energy of $E=50 \mathrm{AMeV}$ for $\mathrm{MD}$ (left) and MI (right) interactions, and for the stiff (solid lines) and soft (dashed lines) iso-EoS, respectively. PT denote particles transferred from the projectile to the target (triangle up, full), and vice versa for TP (triangle down, empty).

- The impact parameter dependence clearly shows that iso-EoS effects are more relevant for more dissipative collisions, i.e. for smaller impact parameters and thus for longer interaction times. This suggests the use of an event selection in terms of the total kinetic energy loss in order to enhance the sensitivity to the symmetry energy, as discussed in Sect.4.3.

Next we note the differences when using the MD or MI interactions in the isoscalar sector. It is seen that the gas is generally more symmetric for a MD interaction. The MD interaction has a stronger isospin-blind repulsion, which emits protons and neutron equally. Thus the mass of the emitted gas is about 5 to 10 units higher in the MD case. This reduces the relative effect of the isovector interaction. Correspondingly, the residues are generally more symmetric for the MI relative to the MD case since more of the neutron excess is emitted into the gas. In addition, in the MD case the reaction proceeds more rapidly, because of the greater isoscalar repulsion, thus reducing the interaction time and reducing the amount of isospin equilibration. This is seen clearly in the mixed systems. 
With respect to different iso-EoS's we see that the gas is more asymmetric for the asy-soft case, which is expected because of the higher sub-saturation symmetry energy and thus the larger neutron repulsion. Consequently the residues are more symmetric for the soft iso-EoS both for the symmetric and the mixed systems. Also, the change of the asymmetries of the residues is much larger for the mixed $(H L)$ than for the symmetric systems, indicating that isospin equilibration will be mostly sensitive to the iso-EoS via the transport contributions of the isospin current. In the exchanged particles we see almost no effect for the particles transferred in the direction projectile to target, and an interesting difference in the other direction. This is due to the fact that, in the direction from projectile to target, the larger isospin diffusion effects expected for the asy-soft EoS are counterbalanced by the more neutron-rich emission to the gas, which reduces the projectile asymmetry. On the other hand, diffusion effects act in opposite direction for the $n$-poor source, with a smaller transferred asymmetry from target to projectile in the asy-soft case, also due to the larger neutron emission to the gas. Thus in this direction we do expect a sensitivity to the iso-EoS, as seen in Fig.7.

However, we have to note that generally the changes due to different isovector EoS's are not very large. As we will see below, the imbalance parameter considerably increases the sensitivity, since the transport contributions will be enhanced. In fact, it was shown in ref. [7] that the transport ratios depend mostly on $\left(\beta_{P T}-\beta_{T P}\right)$. i.e. the asymmetry difference of the exchanged nucleons, and less on pre-equilibrium emissions.

Finally in Fig.8 we compare (for the case of MD interactions) the residue asymmetries for incident energies of $50 \mathrm{AMeV}$ (left panel, already shown in Fig.4) and $35 \mathrm{AMeV}$ (right panel). Competing effects are observed, since on one hand the emission to the continuum is lower at the lower energy, leading to less change of the initial asymmetry, and on the other hand, the interaction time is larger, leading to more equilibration in the mixed system.

\subsection{Isospin Imbalance Ratios}

From the asymmetries shown before one obtains the imbalance ratios according to Eq.(8). These measure the amount of isospin equilibration in the mixed relative to the symmetric reaction systems, having lower absolute values for more equilibration.

The results for the imbalance ratios are shown in Fig.9 for the incident energy of $50 \mathrm{AMeV}$ (left panel) and $35 \mathrm{AMeV}$ (right panel), for MI/MD interactions and different iso-EoS choices. Two very clear trends with respect to the amount of equilibration appear: the equilibration is larger $\left(R_{P}\right.$ smaller $)$ both, 


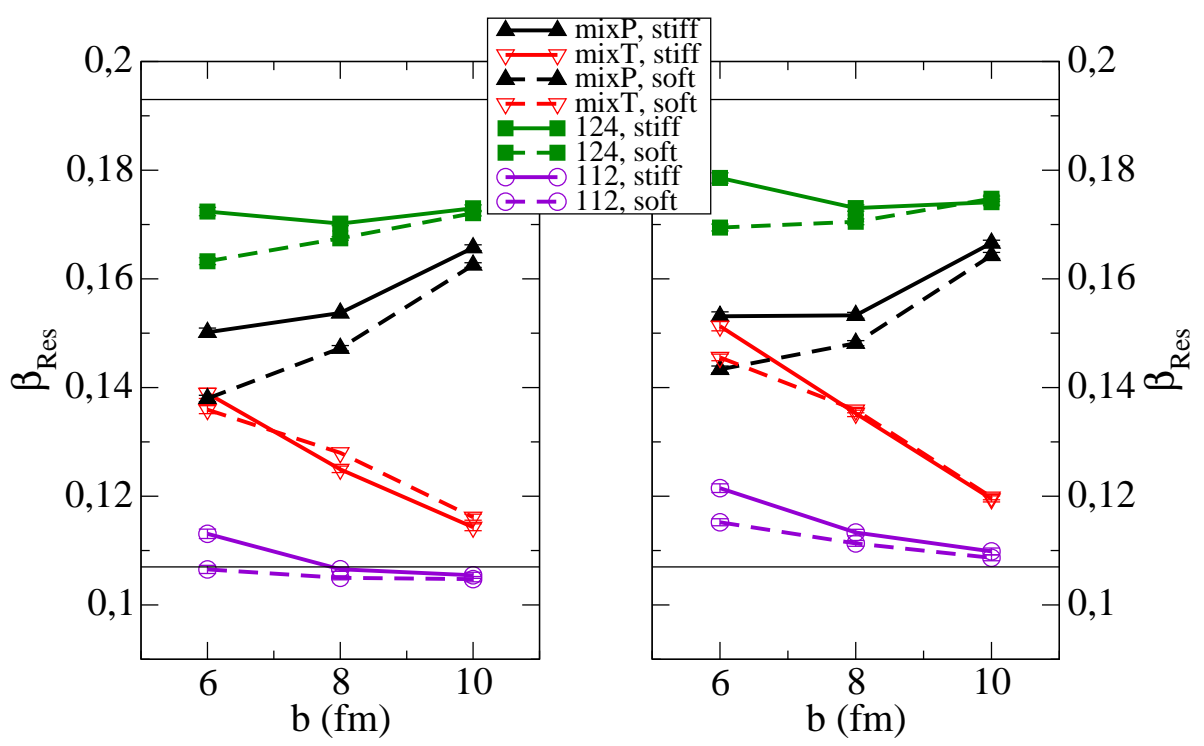

Fig. 8. Asymmetries of the residues in $S n+S n$ collisions at incident energies of $E=50 \mathrm{AMeV}$ (left) and $35 \mathrm{AMeV}$ (right) for MD interactions. The meaning of the lines and symbols is as in Fig.3.

for lower energies (right panel) and for MI interactions (dashed curves). In both cases this can be understood from the fact that the reaction is slower and thus the interaction time longer, leading to more equilibration; for the lower energy because of the lower speed, and for the MI interaction because of the less repulsive isoscalar mean field. In comparing the two iso-EoS's we see that the equilibration is larger for the soft iso-EoS, since the higher symmetry energy leads to a larger diffusion contribution to the isospin current, as discussed above. The first two effects (incident energy and MD interactions) are essentially kinematical, depending on the interaction time, while the last one depends on the iso-EoS, which is of interest here. Thus, in the next section we will propose a scheme to separate the kinematical from the isospin effects.

The results in Fig.9 can be compared to other results in the literature. In our previous work [7] we have used MI interactions and slightly different parametrizations of the iso-EoS, with a somewhat softer symmetry term. The results here agree well with the previous ones within these variations.

On the other hand, there has been extensive discussion of isospin imbalance ratios in several papers of B.A. Li and collaborators [11], in which a different family of iso-EoS's has been used, which is characterized by an index $x$ (for the density dependence of the symmetry energy around saturation of the form $\rho^{\gamma}$ ), together with momentum-dependent interactions, and with free and mediummodified cross sections. The case $x=-1$, corresponding to $\gamma \approx 1$, and with 


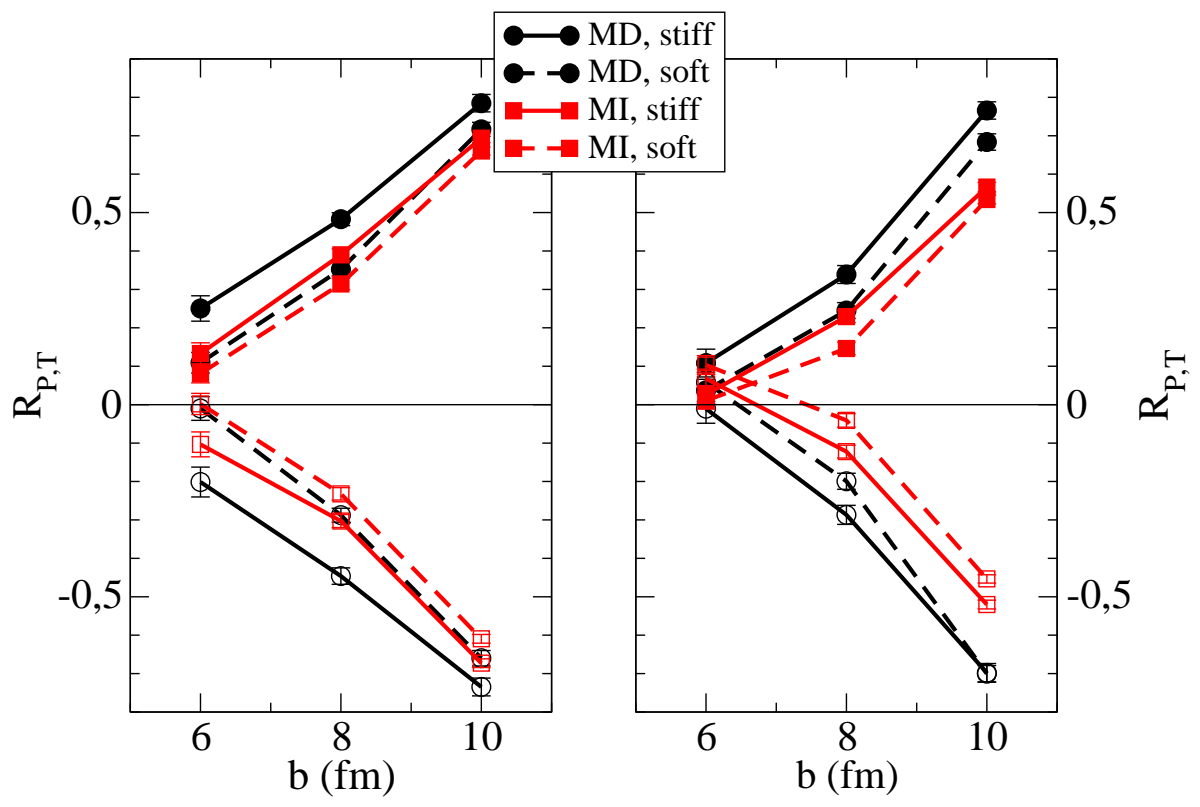

Fig. 9. Imbalance ratios for $S n+S n$ collisions for incident energies of 50 (left) and $35 \mathrm{AMeV}$ (right) as a function of the impact parameter. Signatures of the curves: iso-EoS stiff (solid lines), soft (dashed lines); MD interaction (circles), MI interaction (squares); projectile rapidity ( full symbols, upper curves ), target rapidity ( open symbols, lower curves ).

free cross sections, should be compared with our stiff MD parametrization $(\gamma=1)$. However, the value given in ref. [11] for $b=6 \mathrm{fm}$ of $R_{P} \approx 0.42$ does not agree well with our value of $R_{P} \approx 0.25$ in Fig.9. The reason could reside in ingredients of the BUU transport code used (point test particles, surface effects) leading to a different treatment, relative to the SMF simulations here, of the isoscalar part of the nuclear interaction. This will influence, in turn, the interaction time between the two reaction partners and the degree of dissipation reached in the collision.

The existing imbalance ratio measurements for $S n$ isotopes have been analyzed with an impact parameter selection performed in terms of charged particle multiplicities [8]. A value $R_{P} \approx 0.45$ is extracted in the impact parameter range of $b=6-7.3 \mathrm{fm}$, which is also not far from our asy-stiff MD estimations $(\gamma=1$ or slightly stiffer). From Fig.9 (left panel) we see that in this impact parameter range the imbalance ratio $R_{P}$ is rapidly increasing and so it is very important to assess the same centrality selection in the comparison between data and simulations. To overcome these problems and isolate isospin effects, one could study the imbalance ratio directly as a function of the interaction time (or an observable directly related to it), as we will do in the next section. 


\subsection{Correlation with Kinetic Energy Loss}

In subsection 4.1 we have noted, e.g. in Fig.6, that the residues appear more symmetric for the momentum-independent interaction. Also, we observe in Fig.8 that at lower energy the longer equilibration time is counterbalanced by the smaller pre-equilibrium emission. These trends are more clearly seen in Fig.9 for the imbalance ratio, which directly measures the amount of equilibration. In fact the latter is clearly less effective when either the incident energy is higher or momentum-dependent interactions are used. As we have remarked, both effects decrease the contact time of the two nuclei in the binary collision. Thus these observations point to the interaction time as the dominating influence on the amount of isospin equilibration, as was already discussed in ref. [7].

On the other hand, longer interaction times should be correlated with larger dissipation and, in particular, with damped PLF and TLF velocities. The dissipation, in turn, has been measured, e.g. in deep inelastic collisions, by the kinetic energy loss. Thus in this subsection we will investigate the correlation between the imbalance parameter and the kinetic energy loss. We define the kinetic energy loss per particle as

$$
E_{\text {loss }}=E_{c m}-\frac{E_{k i n}+E_{\text {pot }}^{C o u l}}{A_{P L F}+A_{T L F}}, E_{c m}=\frac{E_{l a b}}{A_{P}} \frac{A_{P} A_{T}}{\left(A_{P}+A_{T}\right)^{2}},
$$

where $A_{P}, A_{T}, A_{P L F}, A_{T L F}$ are the masses of the initial projectile and target, and of the final projectile-like and target-like fragments, respectively. Here $E_{c m}$ is the initial energy, per nucleon, available in the $\mathrm{cm}$ system. $E_{k i n}$ and

$E_{\text {pot }}^{\text {Coul }}$ are the final kinetic energies of the fragments in the $\mathrm{cm}$ system, and the Coulomb potential energy, respectively. We will use the relative energy loss $E_{l o s s} / E_{C M}$ as a measure of PLF/TLF velocities and thus of the interaction time. The study of isospin equilibration as a function of the heavy residue excitation energy (related to the kinetic energy loss ) was also suggested in ref. [9].

The relative energy loss is shown in Fig.10 (left panel) as a function of impact parameter for the two incident energies and MD, resp. MI interactions. It is seen that this quantity exhibits, generally, a very good correlation with impact parameter, except for the large impact parameters, where eventually all curves will converge to zero. As expected the energy loss at a given impact parameter is larger for lower energies and for MI interactions, because of the longer interaction times.

However, the energy loss consists not only in internal excitation of the residues but also in kinetic energy of the particles emitted to the gas. The mass of the 

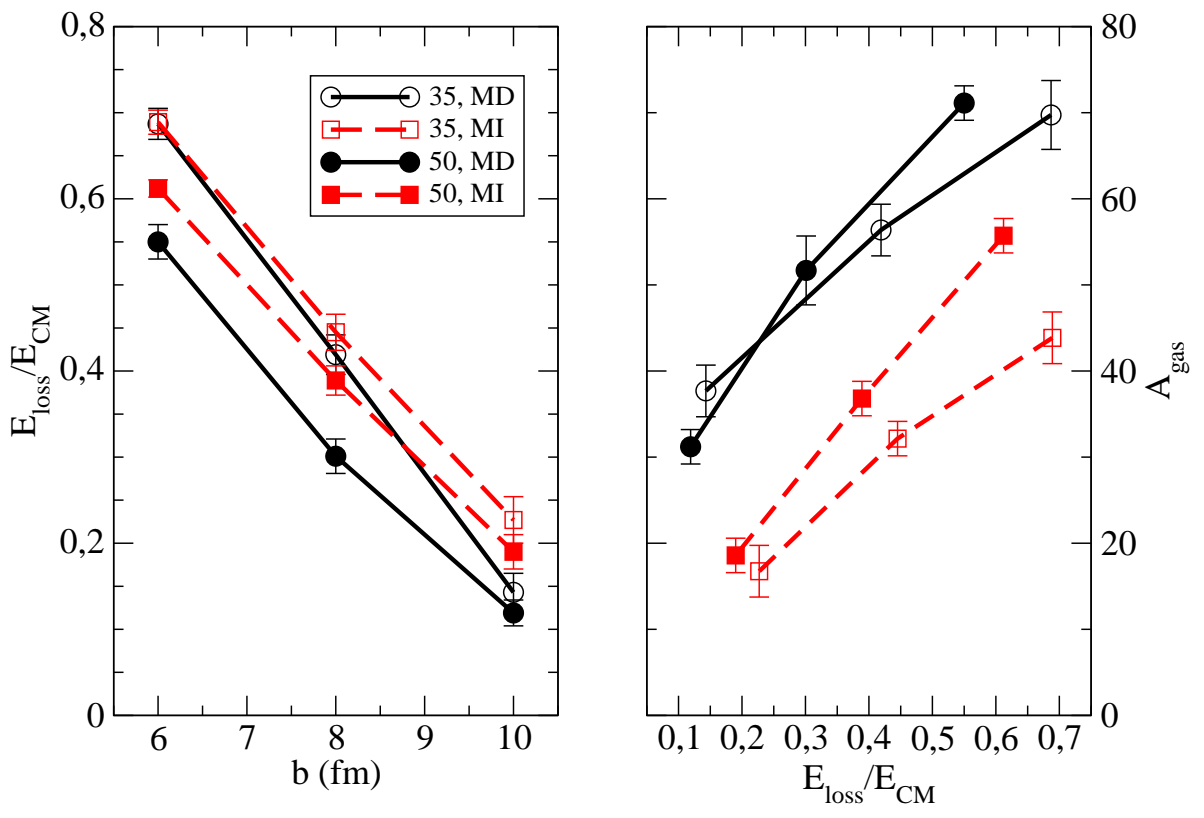

Fig. 10. (left panel) Relative energy loss as a function of impact parameter; and (right panel) mass of particles emitted into the gas as a function of relative energy loss. Curves are shown for MD interactions (solid lines and circles) and MI interactions (dashed lines and squares), and for $35 \mathrm{MeV}$ (open symbols) and $50 \mathrm{MeV}$ (full symbols) incident energy, respectively.

gas is shown in the right hand panel of Fig.10 as a function of the relative energy loss. One sees that the mass loss (and correspondingly the energy loss) to the gas is relatively independent of incident energy for MD interactions (black curves), while there is a larger difference for the MI interactions (grey/red curves). Since the energy lost into the gas is not necessarily closely related to the interaction time, this represents a correction to the correlation between interaction time and energy loss, which is discussed below.

In Fig.11 we present the imbalance ratio as a function of the relative energy loss. In the upper panel we separate the results for MD and MI interactions and stiff and soft iso-EoS, but in each case the results for 35 and $50 \mathrm{MeV}$ are collected together and connected by lines. In contrast to Fig.9 we now see that the points for the different incident energies approximately fall on one line, however with considerable scatter around it. The deviations from a smooth behaviour are larger for the MI interactions (diamonds and triangles). This can be traced back to the observation, made above, that in this case the pre-equilibrium emission, i.e. the energy loss to the gas, is rather different for the two beam energies, while this is not so much the case for the MD interaction. Considering that the pre-equilibrium emission is not a measure of 


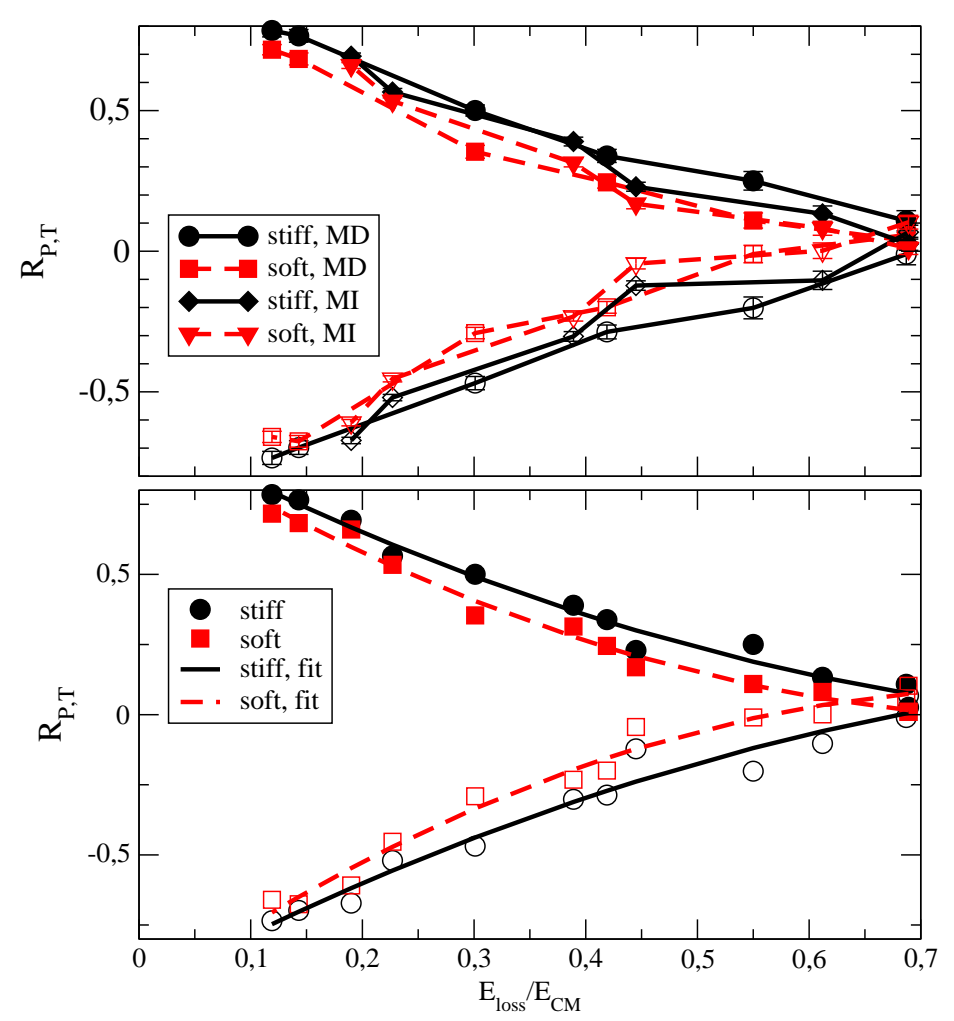

Fig. 11. Imbalance ratio as a function of relative energy loss. Upper panel: Separately for stiff (solid) and soft (dashed) iso-EoS, and for MD (circles and squares) and MI (diamonds and triangles) interactions, in the projectile region (full symbols) and the target region (open symbols). Lower panel: Quadratic fit to all points for the stiff (solid), resp. soft (dashed) iso-EoS.

the interaction time, this introduces a correction to the values obtained for 35 $\mathrm{MeV}$ relative to those at $50 \mathrm{MeV}$, which tends to make the dependence more smooth. We do not attempt, however, to make this correction quantitative, because this would amount to separating the gas particles into pre-equilibrium and evaporation components.

It is seen in Fig.11 that the curves for the asy-soft EoS (dashed) are generally lower in the projectile region (and oppositely for the target region), i.e. show more equilibration, that those for the asy-stiff EoS. In order to emphasize this trend we have, in the lower panel of the figure, collected together all the values for the stiff (circles) and the soft (squares) iso-EoS, and fitted them by a quadratic curve. It is seen that this fit gives a good representation of the trend of the results.

The difference between the curves for the stiff and soft iso-EOS in the lower panel then isolates the influence of the iso-EoS from kinematical effects associated with the interaction time. It is seen, that there is a systematic effect of the symmetry energy of the order of about 20 percent, which should be measurable. The correlation suggested in Fig.11 should represent a general feature 
of isospin diffusion, and it would be of great interest to verify experimentally. As discussed above, this kind of analysis would help also in the comparison with the results of other theoretical models.

On the other hand, it is also seen that the effect is not extremely large, in view also of the substantial scatter of the points around the curves. Thus it is still of interest to investigate other observables, which might show more sensitivity to the iso-EoS. This will be done in the next section with respect to ternary events.

\section{$5 \quad$ Ternary Reactions}

\subsection{Characterization of Intermediate Mass Fragments}

As discussed above, and seen in the example of Fig.3, there are peripheral events in which a third intermediate mass fragment (IMF) appears. The term "neck fragmentation" has been coined for such events, see [35] and refs. therein. Related isospin transport studies have been performed theoretically for symmetric reactions [7] and theoretically and experimentally for asymmetric collisions $[31,36]$. The asymmetry and the kinematical properties of the IMF were shown to carry additional information on the symmetry energy. Here we investigate this information more in detail in the system under study.

In such reactions the asymmetry of the IMF is of particular interest. In symmetric systems it is a result of isospin migration, due to density gradients between the residues and the dilute neck. In mixed systems this effect is in competition with the isospin diffusion discussed in the last section due to concentration gradients. While the study of this competition may yield further information on the time scales of these different processes, the more pure case will be the study of the symmetric systems.

We start with characterizing the origin of the IMF's. As an example in Fig.12

we display various properties of IMF's from the mixed reaction ${ }^{124} S n+{ }^{112} S n$ at $50 \mathrm{AMeV}$ and an impact parameter of $b=6 \mathrm{fm}$ for the stiff (solid histogram, black symbols) and soft (dotted, grey/red ) iso-EoS. In the upper left panel the charge distribution is displayed, exhibiting a broad distribution around charge $Z \approx 8$, which is not very different for different iso-EoS's. The upper right panel shows the times at which the third fragments are identified in our method. It is seen that they appear rather late and in a rather short time span. The lower left panel displays the asymmetry of the IMF's as a function of their charge (later we will only show the average asymmetry). This asymmetry distribution is rather flat; it is, however, substantially different depending on 

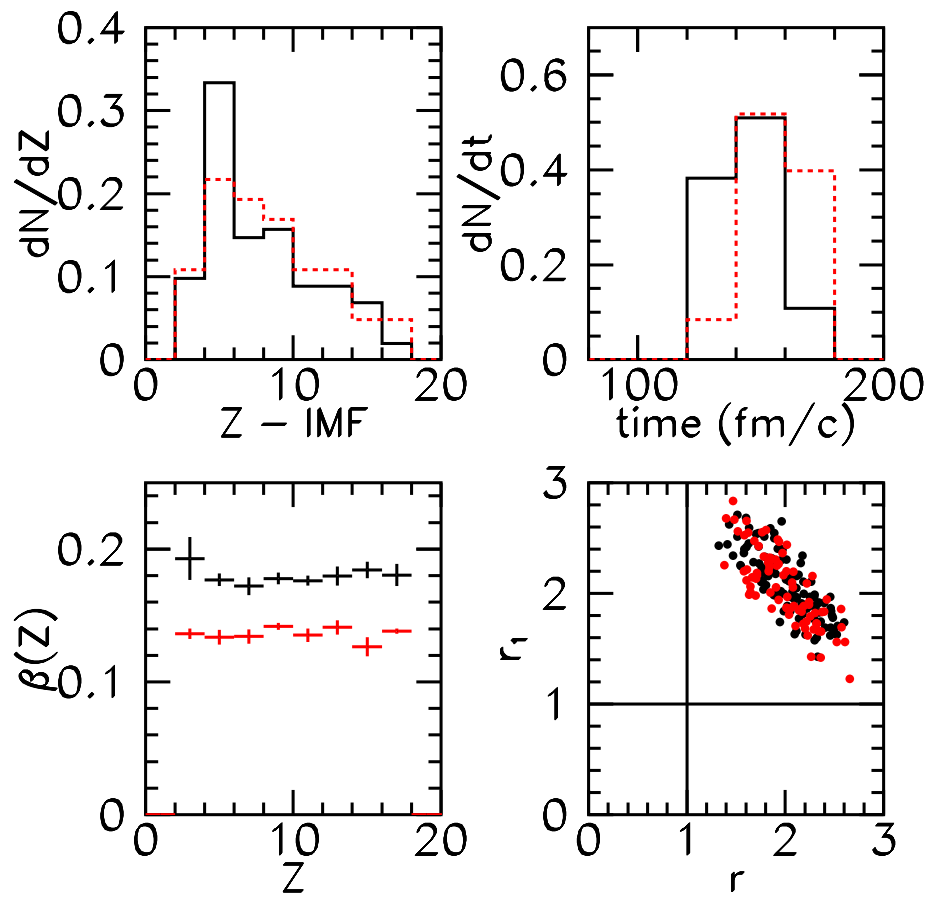

Fig. 12. Properties of Intermediate Mass Fragments (IMF) in the reaction ${ }^{124} \mathrm{Sn}+{ }^{112} \mathrm{Sn}$ at $50 \mathrm{AMeV}$ and $b=6 \mathrm{fm}$ for the stiff (solid lines and black symbols) and soft (dotted lines, grey/red symbols) iso-EoS: (upper left) charge distribution; (upper right) time of appearance of IMF; (lower left) asymmetry distribution of IMF's; (lower right) velocity correlation of IMF with respect to PLF $(r)$ and TLF $(r 1)$, where $r$ is the ratio of the relative IMF-residue velocity to the Viola systematics velocity.

the iso-EoS [30]. Finally in the lower right panel we show velocity correlations between the fragments and the residues, as it was suggested in ref. [31] (socalled Wilczynski-2 plot). The quantity $r$ is the ratio of the relative velocity of the IMF and the PLF to the Viola systematics velocity, which corresponds to fission-like events, and $r 1$ is the same quantity with respect to the TLF. A value of 1 signifies a late, statistical origin of the IMF, while values substantially different from one indicate an IMF of dynamical origin, which is clearly the case here. Altogether the information in this figure then suggests that the IMF's come from a uniform source, which can be identified with the neck region.

\subsection{Isospin Dynamics with Fragment Production}

We have looked at the asymmetries of the various reactions parts (gas, residues and exchanged particles, like in Figs.5, 6 and 7) also for the ternary events. It could be expected that these are different from those of binary events, 


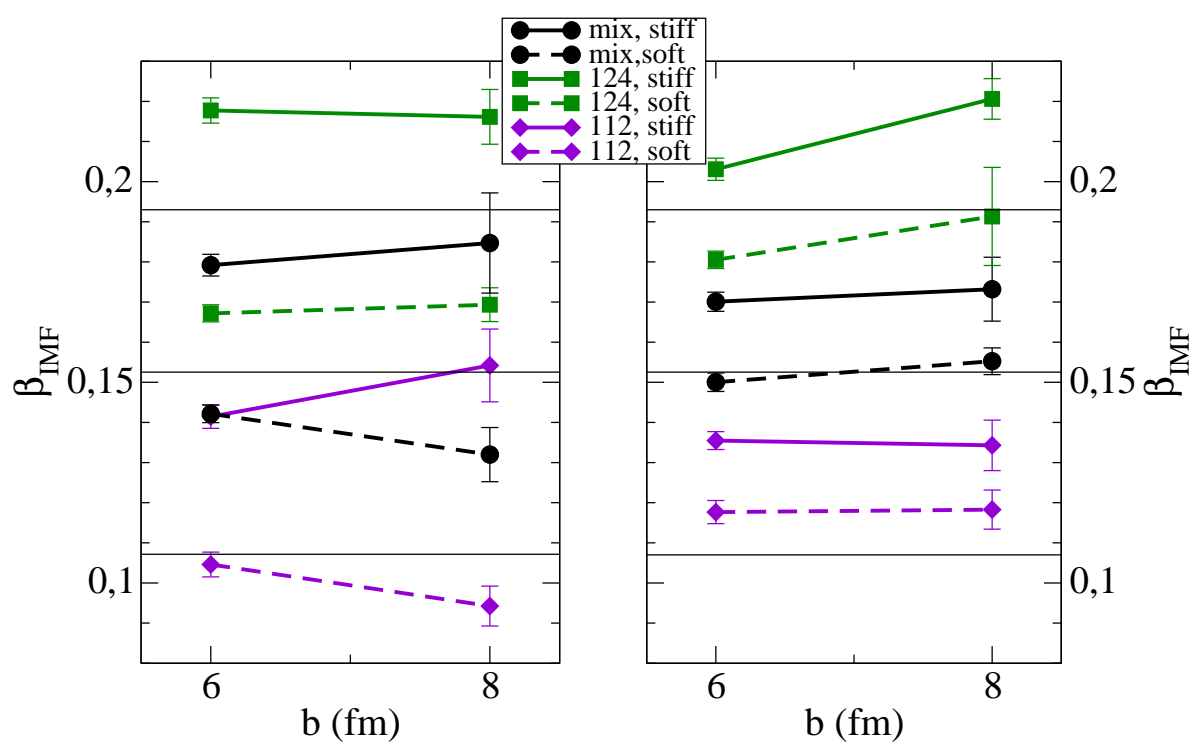

Fig. 13. Asymmetries of IMF's in ternary $S n+S n$ reactions at $50 \mathrm{AMeV}$ as a function of impact parameter for MD (left panel) and MI (right panel) interactions: symmetric $124+124$ (squares), symmetric $112+112$ (diamonds), mixed $124+112$ (circles); stiff iso-EoS (solid lines), soft iso-EoS (dashed lines). Horizontal thin lines denote the asymmetries of ${ }^{124} S n$, mixed reaction and ${ }^{112} S n$, respectively.

because some of the isospin is carried away by the IMF. However, we find that the differences are very small and thus we do not show these figures separately. The relatively small mass of the IMF's thus does not substantially influence the asymmetry of the main reaction products.

A quantity which is very sensitive to the iso-EoS is the asymmetry of the IMF. It is shown in Fig.13 at $50 \mathrm{AMeV}$ for the MD (left panel) and MI (right panel) interactions, separately for the symmetric and the mixed reactions. The asymmetry of the IMF is larger, i.e. the IMF is more n-rich, for the stiff relative to the soft iso-EoS, since the former exhibits a larger isospin migration due to the larger slope of the symmetry energy below saturation. This is clearly the case for the symmetric reactions, but it is also true for the mixed reactions, where there is a competition with isospin diffusion, which depends on the value of the symmetry energy and it is larger for the soft iso-EoS. Our result then shows that the isospin migration is the dominating effect for the asymmetry of the neck fragments.

One may now think to investigate the imbalance ratio for the asymmetry of the IMF, i.e. the ratio in Eq.(8) where the quantity $x$ represents the corresponding average asymmetry of the IMF's independent of their rapidity. As was already seen in ref. [30], this quantity is always found to be close to zero. This means that the asymmetry of the IMF in the mixed reaction is the average of that in the symmetric reactions. This is expected around mid-rapidity for symmetry reasons, although the IMF-asymmetries for all the systems, $H H, L L$ and 
$H L$, are different from the initial values. This is then approximately still true for all the neck fragments. In fact we note that the IMF asymmetries for all systems are changing in the same direction, increasing with the stiffness of the symmetry term. This is a non-trivial condition for the almost zero imbalance ratio of the neck fragments.

However, a very striking feature seen in Fig.13 is the fact that the difference between the IMF-asymmetry for the stiff and the soft iso-EoS's is large, and in addition, that it is much larger in the case of MD interactions relative to MI interactions (difference between solid and dashed lines in the two panels, for each colliding system). The large sensitivity of the IMF asymmetry to the iso-EOS was already noted earlier [30] for MI interactions, showing the effectiveness of isospin migration for a stiff iso-EoS.

An interesting point which deserves a deeper interpretation is that the iso-EoS effect appears even more prominent for MD interactions. We see that $\beta_{I M F}$ increases for the asy-stiff case (solid lines) and decreases for the asy-soft one (dashed lines) for MD relative to MI interactions. Therefore the differences in the predicted IMF-asymmetries for different iso-EoS's are systematically enhanced. This can be traced back to the following effect of MD interactions: the reaction is faster and so we obtain a mid-rapidity neck fragment only in regions with larger projectile-target overlaps. Thus, for a given impact parameter the IMF is produced in a more compact configuration, i.e. with a larger interface with the spectators [37] near normal density, where the asy-stiff EoS is effective with respect to neutron migration to the neck, while the asy-soft is not, due to the low value of the derivative of the symmetry energy close to normal density (see Fig.1). In conclusion, with MD interactions we see a more prominent iso-EoS effect with a strong $n$-enrichment of the neck fragments only for the asy-stiff case.

\subsection{Asymmetry Ratios}

It is of interest to construct an observable, which quantifies these effects and which does not depend sensitively on measuring the absolute asymmetries, which are changed by the secondary evaporation. Considering that the asymmetries of the other reaction products (residues and the gas) is not equally sensitive to the iso-EoS (see Figs. 5 and 6 ; which are for binary events, but, as stated above, do not change noticeably for ternary events), it is attractive to look for ratios of asymmetries. In Fig.14 we show the ratio of the asymmetries of the IMF's to those of the residues for the symmetric $S n+S n$ reactions for stiff (solid) and soft (dashed) iso-EoS, and for MD (circles) and MI (squares) interactions. The results correspond to $b=6 \mathrm{fm}$ semiperipheral events, plotted here as a function of the initial isospin asymmetry of the colliding system. 


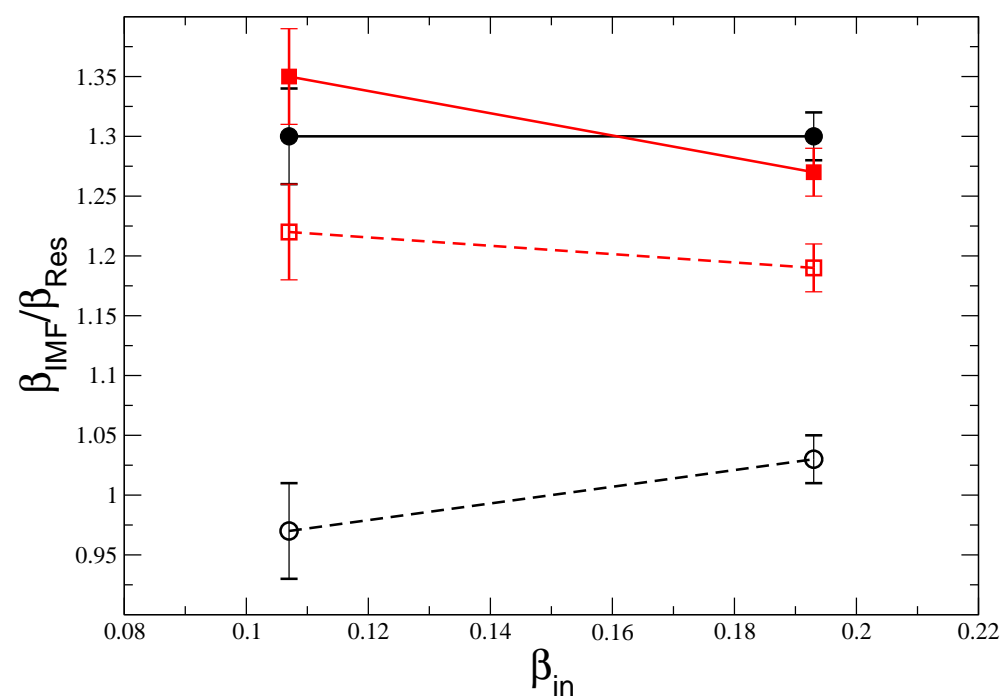

Fig. 14. Ratio of asymmetries of IMF to residues for symmetric $S n+S n$ reactions as a function of the initial isospin asymmetry for semiperipheral events $(b=6 \mathrm{fm})$ at $50 \mathrm{AMeV}$ beam energy. Results are shown for MD (circles) and MI (squares) interactions, and for stiff (solid lines) and soft (dashed lines) iso-EoS's.

The ratio between the asymmetry of IMF's and residues can be estimated on the basis of simple energy balance considerations. In fact, isospin migration is due to the fact that the neck region has lower density with respect to the residues and the symmetry energy is decreasing with density. Starting from a residue of mass $A_{\text {res }}$ and a neck of mass $A_{I M F}$ of different density but uniform asymmetry $\beta$, we assume that the mass $A$ participating in the isospin exchange is approximately equal to the mass of the neck, while it is small relative to the mass of the residue. This will lead to the asymmetry $(\beta+\Delta \beta)$ of the neck, and to a total asymmetry $\beta_{\text {res }}=\left[\beta\left(A_{\text {res }}-A\right)+(\beta-\Delta \beta) A\right] / A_{\text {res }}=\beta-\Delta \beta A / A_{\text {res }}$ of the residue, with $\Delta \beta$ to be determined by minimization of the symmetry energy. The corresponding variation of the symmetry energy is equal (apart from a constant) to:

$$
\Delta E_{\text {sym }}=A_{\text {res }} E_{\text {sym }}\left(\rho_{R}\right)\left(\beta-\Delta \beta A / A_{\text {res }}\right)^{2}+A E_{\text {sym }}\left(\rho_{I}\right)(\beta+\Delta \beta)^{2},
$$

where $\rho_{R}$ and $\rho_{I}$ are the densities of the residue and neck regions, respectively. The minimum of the variation of $\Delta E_{\text {sym }}$ yields

$$
\frac{\beta_{I M F}}{\beta_{\text {res }}}=\frac{E_{\text {sym }}\left(\rho_{R}\right)}{E_{\text {sym }}\left(\rho_{I}\right)}=1+\frac{E_{\text {sym }}\left(\rho_{R}\right)-E_{\text {sym }}\left(\rho_{I}\right)}{E_{\text {sym }}\left(\rho_{I}\right)}
$$

From this simple argument the ratio between the IMF and residue asymmetries should depend only on symmetry energy properties and, in particular, on the difference of the symmetry energy between the residue and the neck regions, as appropriate for isospin migration. It should also be larger than one, more 


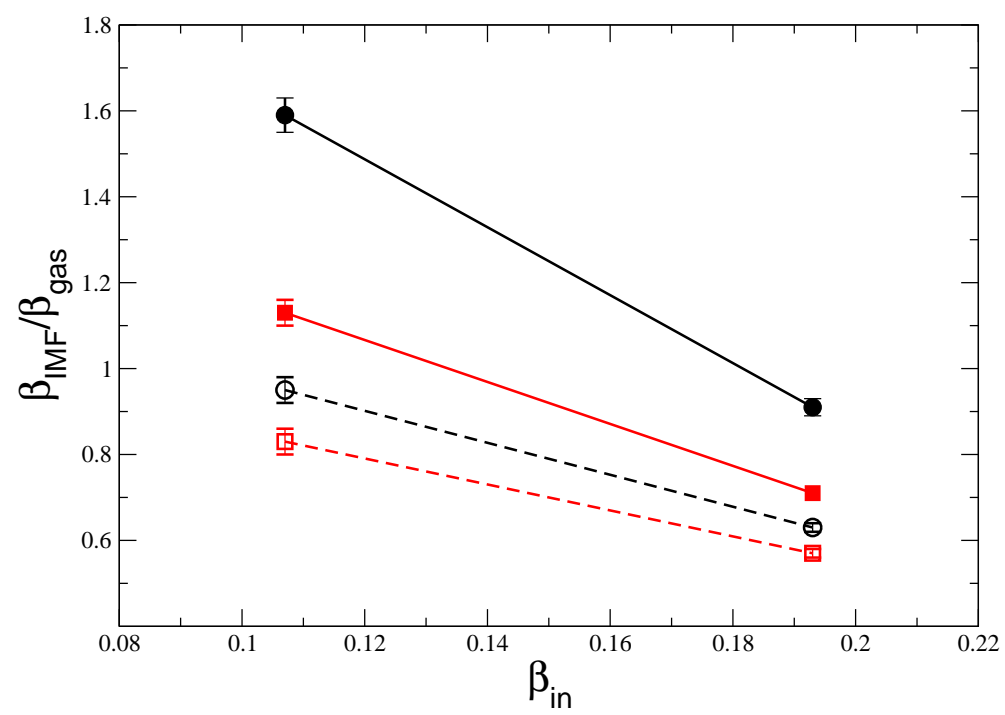

Fig. 15. Ratio of asymmetries of IMF to gas for symmetric $S n+S n$ reactions as a function of the initial isospin asymmetry. Semiperipheral events $(b=6 \mathrm{fm})$ at $50 \mathrm{AMeV}$ beam energy. Signatures are as in Fig. 12

so for the asy-stiff than for the asy-soft EoS.

It is seen indeed in Fig.12, that this ratio of IMF over residues asymmetry is nicely dependent on the iso-EoS only and not on the initial asymmetry. This is a clear feature of the dominant isospin migration mechanism which depends only on the density gradients. The ratio is also mostly larger than unity. In both cases, MI and MD interactions, the asy-stiff EoS is more effective in the nenrichment of the dilute region where the IMF's are produced. Moreover, since for the asy-stiff EoS, the derivative of the symmetry energy with respect to the density is constant, isospin migration effects should be essentially the same in MI and MD interactions, as roughly confirmed by Fig.12. On the other hand, isospin effects appear quite reduced for the asy-soft EoS, especially for the MD interaction for the reasons already mentioned (reduced slope and larger neutron emission for the asy-soft EoS for the more compact configurations of the MD dynamics). These behaviours leads to a rather large sensitivity to the iso-EOS for the more realistic MD interactions. We note that the iso-EoS effect amounts to more than 30\%. Since we expect the secondary evaporation to affect in a similar way the asymmetry of IMF's and residues, we suggest this observable as very sensitive probe to the isovector part of the EoS.

A similar investigation can be performed with the ratio of the asymmetries of the IMF to those of the gas. The corresponding results are shown in Fig.15. The mechanism of the gas emission is not simply related to the isospin migration and now the ratios are seen to be more dependent of the initial asymmetry. The decreasing trend seen from ${ }^{112} S n$ to ${ }^{124} S n$ collisions is a consequence of the larger asymmetry of the gas for the more isospin asymmetric systems (see Fig. 5). Impressive is the large value of this ratio for the ${ }^{112} \mathrm{Sn}$ collisions and 
MD interaction and asy-stiff EoS, which nicely confirms the interpretation of a larger proton enrichment and thus smaller asymmetry of the gas phase. Also for this observable a larger sensitivity to the iso-EoS is observed in the $\mathrm{MD}$ case. This reflects the large difference in the predicted IMF asymmetry between the two iso-EoS's, as discussed above.

\subsection{Double Ratios}

In order to further reduce the secondary decay effects it has recently been proposed to look at double ratios of isospin-dependent quantities, $n$-rich vs. $n$-poor systems, e.g. in central collisions for the asymmetries of nucleon [19] or IMF [20] emissions. From Figs.14/15 this double ratio should always be around unity for $\beta_{I M F} / \beta_{\text {Res }}$, not much depending on the initial asymmetry, while it appears of interest for the quantity $\beta_{I M F} / \beta$ gas. From the same class of semiperipheral events we can extract Tab.III for the Double Ratios:

$$
\frac{\beta_{I M F} / \beta \operatorname{gas}\left({ }^{124} S n+{ }^{124} S n\right)}{\beta_{I M F} / \beta \operatorname{gas}\left({ }^{112} S n+{ }^{112} S n\right)}
$$

We note the almost $20 \%$ Iso-EoS dependence for the more realistic $M D$ interactions.

Tab. III: $\beta_{I M F} / \beta$ gas Double Ratio.

\begin{tabular}{c|c|c}
\hline Interaction & $\mathrm{MD}$ & $\mathrm{MI}$ \\
\hline Asy-soft & 0.660 & 0.687 \\
\hline Asy - stiff & 0.572 & 0.628 \\
\hline
\end{tabular}

\section{Summary and Perspectives}

In this work we have systematically studied isospin transport in heavy ion collisions in the Fermi energy domain for symmetric and asymmetric combinations of $S n$ isotopes. We have focused on semi-peripheral dissipative collisions to study both isospin equilibration between the residues and fragmentation of the neck between the residues, i.e. to study the isovector part of the EoS for densities below saturation density. The purpose of this investigation has been to analyze in detail the mechanisms of isospin dynamics and to identify observables, which are sensitive to the still controversial iso-EoS. We have employed two typically different iso-EoS's (asy-soft and asy-stiff), where one expects to see characteristic signatures since the value and the density-slope of 
the symmetry energy determine the effects of isospin migration (due to density differences) and isospin diffusion (due to isospin concentration differences).

We have clearly shown that the isospin transport is also dependent on the overall reaction dynamics, mostly ruled by the isoscalar properties of the effective interactions. Thus, it is important to select observables able to disentangle between isoscalar and isovector contributions.

In this respect we have thoroughly analyzed the effects of the well established (isoscalar) momentum dependence of the mean field on isospin transport phenomena. The momentum dependence of the effective forces can affect the relationship between isospin transport observables and the symmetry energy since the reaction dynamics is modified. In particular, the interaction times are shorter and the composite nuclear systems break starting from more compact configurations. It is then difficult to distinguish effects of the iso-EoS and of the momentum-dependence in the usual analysis as a function of centrality.

For binary dissipative reactions we therefore suggest a study of the imbalance ratios as a function of the relative energy loss, which is in turn well correlated to the interaction time. We thus obtain rather well defined universal curves, including results from different MD interactions at different beam energies, which present, however, a clear dependence on the iso-EoS of the order of $20 \%$. This kind of analysis should be useful and illuminating in the comparison with experimental data, as well as in testing the predictions of different theoretical models. In fact, it allows to disentangle isospin effects from other model ingredients that may influence the degree of dissipation reached in the system.

For ternary events, where an Intermediate Mass Fragment (IMF) is formed in the mid-rapidity region (neck fragmentation), the IMF neutron enrichment is very sensitive to the iso-EoS. We have isolated the isospin migration as the dominant mechanism in this process in collisions of the symmetric systems $\left({ }^{124} S n+{ }^{124} S n\right.$ vs. $\left.{ }^{112} S n+{ }^{112} S n\right)$. The iso-EoS dependence is clearly emerging, being stronger for an asy-stiff choice with a larger slope of the symmetry energy. We have also found that the effect is larger for the more realistic MD dynamics, due to different density regions which are probed.

All the properties of the reaction products, i.e. residues, gas and IMF's, are evaluated at the freeze-out time and thus they can be modified by the sequential decay of the excited products. We therefore propose the investigation of "relative" isospin contents, i.e. of ratios of the asymmetry $\beta=(N-Z) / A$ of the various products, which are expected to be less affected by secondary emissions. A very promising quantity seems to be the ratio $\beta_{I M F} / \beta_{\text {Res }}$ at midcentrality, which directly measures the isospin migration. In fact, it is found to be very sensitive to the iso-EoS, up to above the $30 \%$ level for the more 
realistic $\mathrm{MD}$ interactions.

In conclusion, we suggest new isospin sensitive observables to study in dissipative HIC at Fermi energies in order to further constrain value and slope of the symmetry term at subsaturation densities.

It should also be noticed that the ratios considered here (Imbalance Ratios, $\left.\beta_{I M F} / \beta_{\text {Res }}\right)$ do not depend, at a first level approximation, on the initial asymmetry of the systems considered, but only on the properties (value and slope) of the symmetry energy. Hence valuable information on these properties can already be obtained with the available neutron-rich beams. However, the use of radioactive beams in this intermediate energy range would be certainly important in order to extend the systematics of results and to better constrain the symmetry energy behaviour.

\section{Acknowledgments}

We warmly thank M.B.Tsang for fruitful discussions. This work was supported partly by the German Ministry for Education and Research (BMBF), grant 06LM189, and by the DFG Cluster of Excellence Origin and Structure of the Universe (www.universe-cluster.de). V.B. acknowledges the support of the Romanian Minister for Education and Research, under the contracts No.CEX05-D10-02 and CEX-05-D11-03. V.B., H.H.W. and M.Z.-P. are grateful to the LNS-INFN for the warm hospitality during part of this work.

\section{References}

[1] M. Colonna, M. Di Toro, G. Fabbri, S. Maccarone, Phys. Rev. C57 (1998) 1410

[2] B.A. Li, C.M.Ko, W. Bauer, Int. J. Mod. Phys. E7 (1998) 147

[3] Isospin Physics in Heavy-ion Collisions at Intermediate Energies, Eds. B.A. Li and W. Udo Schröder, Nova Science Publishers (2001, New York)

[4] V. Baran, M. Colonna, V. Greco, M. Di Toro, Phys. Rep. 410 (2005) 335

[5] M.Colonna and M.B.Tsang, Eur. Phys. J. A30 (2006) 165, and refs. therein.

[6] C.Fuchs, H.H.Wolter, (WCI book) Eur. Phys. Jour. A30 (2006) 5, and refs. therein.

[7] V. Baran, M. Colonna, M. Di Toro, M. Zielinska-Pfabe, H.H. Wolter, Phys. Rev. C 72 (2005) 064620

[8] M.B. Tsang, et al., Phys. Rev. Lett. 92 (2004) 062701 
[9] G.A. Souliotis, M. Velselsky, D.W. Shetty, S.J. Yennello, Phys. Lett. B588 (2004) 35

[10] L. Shi, P. Danielewicz, Phys. Rev.C68 (2003) 064604

[11] B.A. Li, L.W. Chen, Phys. Rev. C 72 (2005) 064611;

L.W. Chen, C.M. Ko, B.A. Li, Phys. Rev. Lett 94 (2005) 032701

[12] A.Guarnera, M.Colonna, P.Chomaz, Phys. Lett. B373 (1996) 267

[13] M.Colonna, M. Di Toro, A. Guarnera, S. Maccarone, M. Zielinska-Pfabe, H.H. Wolter, Nucl. Phys. A642 (1998) 449

[14] M. Colonna, G. Fabbri, M. Di Toro, F. Matera, H.H. Wolter, Nucl. Phys. A742 (2004) 337

[15] P.Chomaz, M.Colonna, J.Randrup, Phys. Rep. 389 (2004) 263

[16] A. Guarnera, TWINGO Code, Ph.D. Thesis, Univ. of Caen 1996

[17] J. Rizzo, Collective and Stochastic Observables in HIC at Intermediate Energies Ph.D. Thesis, Univ. Catania 2006

[18] G.Q. Li, R. Machleidt, Phys. Rev. C48 (1993) 1702; Phys. Rev. C49 (1994) 566

[19] M.A. Famiano, et al., Phys. Rev. Lett. 97 (2006) 052701

[20] M. Colonna, V. Baran, M. Di Toro, H.H. Wolter, Isospin Distillation with Radial Flow: a Test of the Nuclear Symmetry Energy, arXiv:0707.3092 (nucl/th)

[21] J. Rizzo, M. Colonna, M. Di Toro, Phys. Rev.C72 (2005) 064609

[22] M. Di Toro, M. Colonna, J. Rizzo, AIP Conf.Proc. 791 (2005) 70-82

[23] C. Gale, G.F. Bertsch, S. Das Gupta, Phys. Rev. C38 (1987) 1666

[24] C. Gale, G.M. Welke, M. Prakash, S.J. Lee, S. Das Gupta, Phys.Rev. C41 (1990) 1545

[25] V.Greco, Master Thesis, Catania Univ. (1997);

V.Greco, A.Guarnera, M.Colonna, M.Di Toro, Phys.Rev. C59 (1999) 810;

Nuovo Cimento A111 (1998) 865

[26] B.-A.Li, C.B.Das, S.Das Gupta, C.Gale, Nucl.Phys. A735 (2004) 563

[27] E.Chabanat, P.Bonche, P.Haensel, J.Meyer, R.Schaeffer, Nucl.Phys. A627 (1997) 710

[28] E.Chabanat, P.Bonche, P.Haensel, J.Meyer, R.Schaeffer, Nucl.Phys. A635 (1998) 231

[29] P. Danielewicz, R. Lacey, W.G. Lynch, Science 298 (2002) 1592

[30] V. Baran. M. Colonna, M. Di Toro, V. Greco, M. Zielinska-Pfabe, H.H. Wolter, Nucl. Phys. A 703 (2002) 603 
[31] V. Baran, M. Colonna, M. Di Toro, Nucl. Phys. A730 (2004) 329

[32] F. Rami et al., Phys. Rev. Lett. 84 (2000) 1120

[33] V.Baran, D.M.Brink, M.Colonna, M.Di Toro, Phys. Rev. Lett. 87 (2001) 182501

[34] M.Di Toro, M.Colonna, C.Rizzo and V.Baran, The Dinamical Dipole Radiation in Dissipative Collisions with Exotic Beams, arXiv:0711.3535[nuclth], Int.Jou.Modern Physics E17 (2008) in press.

[35] M. Di Toro, A. Olmi, R. Roy, (WCI book) Eur. Phys. Jour. A30 (2006) 65, and refs. therein.

[36] E. De Filippo, et al. (Chimera Coll.), Phys. Rev. C 71 (2005) 044602

[37] J. Rizzo, P. Russotto, IMF formation in 64Ni+124Sn peripheral collisions at $35 \mathrm{AMeV}$, Ph.D. School internal report, Univ. di Catania, 2006 